\title{
The role of the central stellar cluster in active galactic nuclei
}

\section{Semi-analytical model}

\author{
E. Y. Vilkoviskij ${ }^{1,2}$ and B. Czerny ${ }^{3}$ \\ 1 Fesenkov Astrophysical Institute, Observatory, 480020 Almaty, Kazakstan \\ e-mail: vilk@afi.south-capital.kz \\ 2 Isaac Newton Institute of Chile, the Kazakhstan branch \\ 3 Copernicus Astronomical Center, Bartycka 18, 00-716 Warsaw, Poland
}

Received 29 August 2001 / Accepted 18 February 2002

\begin{abstract}
The subject of the paper is the role of the massive stellar cluster in the activity phenomenon and in the structure of active galactic nuclei. We introduce a simple model of stellar dynamics in the internal part of the cluster, which allows us to include both the star-disk and the star-star interactions. It is shown that the properties of the distribution of stars in the vicinity of the black hole are determined both by the interaction of the stars with the accretion disk and by the pair gravitational and contact interaction between the stars. We calculate the distribution of stars in the central parts of the cluster and we discuss possible effects of stellar mass-loss due to the star-disk interaction. Finally, we study the implications of the central cluster for active galactic nuclei activity. We model the broad line region assuming that the gaseous wakes, following stars after each disk crossing, play the role of the broad line region clouds, and we calculate the corresponding line profiles. We also analyze the contribution of star-star and star-disk collisions to active galactic nuclei variability.
\end{abstract}

Key words. galaxies: active - galaxies: Seyfert - stellar dynamics - ultraviolet: galaxies - X-rays: galaxies

\section{Introduction}

The idea of an important role of the compact stellar cluster (hereafter CSC) in creation and evolution of active galactic nuclei (hereafter AGN) was formulated at the very early stage of AGN investigation (e.g., Shklovskiy 1964), and the evolution of a gas sphere containing a CSC was first considered in the work of Unno (1971). The dissipative interaction of the compact stellar cluster with the massive central object in the form of a super-star was considered by Vilkoviskij (1975) and Hara (1978), and the tidal interaction of stars with the central object in the form of a massive black hole (hereafter $\mathrm{MBH}$ ) by Hills (1975). The evolution of the dense, non-rotating stellar cluster was discussed by Saslaw (1966), Bisnovatyi-Kogan (1978), and others. The interaction of stars with the accretion disk (hereafter AD) was considered in the works by Vilkoviskij \& Bekbosarov (1981), Vilkoviskij (1983), Zentsova (1983), Syer et al. (1991). More detailed investigations of the evolution of the stellar orbits crossing the AD were presented in Artymowicz et al. (1993), Karas \& Vokrouhlicky (1993), Rauch (1995), Vokrouhlicky \& Karas (1993, 1998), Subr \& Karas (1999), and others. The role

Send offprint requests to: B. Czerny,

e-mail: bcz@camk.edu.pl of the outer parts of $\mathrm{AD}$ (which can be gravitationally unstable) in the global evolution of AGNs through efficient star formation was discussed by Collin \& Zahn (1999).

A general investigation of the problems of CSC structure and evolution, taking into account the interaction of the star cluster with the accretion disk, demands calculations of very complicated numerical models. In contrast, the main aim of the present paper is a preliminary analysis at a qualitative level, but including both star-star and star-disk interactions. The star-disk and the star-star interactions were not considered before as acting simultaneously. Rauch $(1995,1999)$ considered and numerically calculated the cases when either star-disk or star-star collisions dominate; we consider here a model which is very simple but includes the joint action of the star-disk and star-star interactions. This permits us to find the space distribution of the stars around ADs. As an application of this result, we consider star-related model of Broad Emission Line Regions (hereafter BELRs) and the possible role of the inelastic interactions of stars both with the disk and other stars in the variability of AGNs.

The plan of the paper is as follows. In Sect. 2 we consider the stellar dynamics in the central part of CSC in AGNs. In Sect. 3 we derive the structure of the stellar cluster in the $\mathrm{AD}$ region, including the role of contact stellar 
collisions and some effects of the mass loss of the stars. The application of the results to modeling BELR and variability are discussed in Sects. 4 and 5, correspondingly. The discussion of the results and conclusions are given in Sect. 6.

\section{Stellar dynamics in the central part of CSC}

\subsection{The star-disk interaction}

We assume that there are massive and compact stellar clusters in the centers of AGNs. Observations of the nearest galaxies show clearly that dense stellar clusters do exist in their centers, with sizes close to a parsec, with masses $10^{7}-10^{8} M_{\odot}$, and central stellar densities $10^{6}-10^{7} \mathrm{pc}^{-3}$. It is reasonable to suppose that in AGNs the density in CSCs is increased, and in the case of the more powerful AGNs the central stellar density can reach values of up to $10^{8}-10^{9} \mathrm{pc}^{-3}$, as follows in particular from the gas-dynamic solutions for the broad absorption line QSOs (hereafter BAL QSO, see Vilkoviskij et al. 1999). In contrast to the case of close galaxies with low activity, the observational data about CSCs in luminous AGNs are very poor; we will assume that those CSCs are massive, rotate, and consist predominantly of low-mass stars.

For the sake of qualitative analysis, we introduce several simplifications in our consideration: (1) we consider equal stellar masses, (2) we assume that all stellar orbits are almost circular, which is a good approximation in the internal part of the CSC only (see below), (3) we introduce some effective values of the main physical parameters the inclination angle of stellar orbits to the plane of $\mathrm{AD}$, and the stellar density (see the next subsection), and we investigate the radial dependence of these parameters.

The star-disk (hereafter also s-d) interactions were considered in a series of studies. The main, and quite obvious result of s-d interaction is that the orbits of stars that have been captured into bound orbits crossing the accretion disk evolve from eccentric to almost circular, with diminishing radius and inclination angle (e.g., Syer et al. 1991). The planes of the orbits approach the plane of the $\mathrm{AD}$ in the process of the orbit evolution. As a result, the stars on these evolved orbits move mostly in the direction of the $\mathrm{AD}$ rotation, due to the dissipative interaction of the stars with the disk. For a single star, this process leads finally to the incorporation of the stellar orbit into the disk plane, and the further fate of such stars is undefined (Artymowicz et al. 1993). In the next sections we will show that consideration of the collective star inflow through the disk instead of the single stellar orbit evolution change the result: due to high stellar density, the star-star interaction scatters stellar orbits, thus counteracting the capture of stars by the disk. This effect allows the CSC to reach some equilibrium, with fluctuating but non-vanishing inclination angle of the orbits, and the dissipative inflow of the stars continues to the center of the AD.

In the present section we will shortly summarize the properties of the individual star-disk interaction. For simplicity we will take into account two above-mentioned basic theoretically deduced properties of the orbits, crossing the disk: we assume that all orbits are close to the circular shape, and that the inclination angles of the orbits to the disk are small.

Let us consider a star in an orbit, crossing the $\mathrm{AD}$ in the zone where the gravity of the MBH dominates. The velocity of a star, crossing the AD at a distance $R$ is

$V_{\mathrm{s}}=q c\left(R_{\mathrm{g}} / R\right)^{1 / 2}=q c\left(10^{-3} m_{8} / r_{-2}\right)^{1 / 2}$,

where $c$ is the velocity of light, $R_{\mathrm{g}}=2 G M_{\mathrm{BH}} / c^{2}$ is the gravitation radius of $\mathrm{MBH}, m_{8}=M_{\mathrm{BH}} / 10^{8} M_{\odot}$ is the MBH mass, $r_{-2}=R /(0.01 \mathrm{pc})$ is the distance from the MBH in units of $0.01 \mathrm{pc}$, and $1>q \geq 1 / \sqrt{2}$ is the coefficient for the Keplerian orbits: $q$ is close to 1 for the very stretched orbits, and $q=1 / \sqrt{2}$ for the circular ones. In our approximation of almost circular orbits we take $q=1 / \sqrt{2}$, and $V_{\mathrm{s}} \simeq \sqrt{G M / R}$. So in convenient units $v_{\mathrm{s} 9}=V_{\mathrm{s}} /\left(10^{9} \mathrm{~cm} \mathrm{~s}^{-1}\right)$,

$v_{\mathrm{s} 9} \simeq 0.67\left(m_{8} / r_{-2}\right)^{1 / 2}$.

The normal and tangential velocity components with respect to the thin disk are $V_{\mathrm{n}}=V_{\mathrm{s}} \sin i$ and $V_{\mathrm{t}}=V_{\mathrm{s}} \cos i$, where $i$ is the orbit-to-disk inclination angle. The gas motion in the disk is also close to the circular one, $V_{\mathrm{d}} \simeq V_{\mathrm{s}}$; so, the relative velocity of the star and gas in the Keplerian disk is $V_{\mathrm{re}}=\left[V_{\mathrm{n}}^{2}+\left(V_{\mathrm{d}}-V_{\mathrm{t}}\right)^{2}\right]^{1 / 2}$, and we obtain $v_{\mathrm{re} 9} \simeq$ $v_{\mathrm{s} 9} 2^{1 / 2}(1-\cos i)^{1 / 2}$. Note that this velocity is supersonic in the disk with $T_{\mathrm{d}} \sim 10^{4} \mathrm{~K}$ even if the inclination angle is very small, down to $i \sim 0.1^{\circ}$.

It is obvious that the drag force acting at the star in the disk is proportional to the cross-section of the star, the disk density, and the square of the relative star-disk velocity, but the coefficient of the proportionality is known with an accuracy of a factor of a few; hereafter we will denote it with $Q$. Several attempts to estimate $Q$ (Zentsova 1983; Ostriker 1983; $\dot{Z}$ urek et al. 1994) gave slightly different results. We will use for the energy loss of the star crossing thin $\mathrm{AD}$ the expression

$\Delta E=Q \pi R_{\mathrm{s}}^{2} \Sigma_{\mathrm{d}}\left(V_{\mathrm{s}}\right)^{2} \phi(i)$

where $R_{\mathrm{s}}$ is the radius of the star, $\Sigma_{\mathrm{d}}$ is the surface mass density of the $\mathrm{AD}$, and $\phi(i)$ is the function of the inclination angle. Within our approximations, one has $\phi(i) \sim\left[\left(V_{\mathrm{n}}\right)^{2}-\left(V_{\mathrm{d}}-V_{\mathrm{t}}\right)^{2}\right] /\left(V_{\mathrm{s}}\right)^{2}=2 \cos i(1-\cos i)=$ $2 \sin i^{2} \cos i /(1+\cos i)$.

Physically this means that the star gives its velocity (in the system co-rotating with disk) to the column of the disk matter with cross-section close to that of the star, and with height equal to the disk thickness. This brakes the normal component of the star velocity, but accelerates the star in the direction of the disk rotation.

The energy generated by the drag force is

$E_{\mathrm{diss}} \sim Q \pi R_{\mathrm{s}}^{2} \Sigma_{\mathrm{d}}\left(V_{\mathrm{re}}\right)^{2}=Q \pi R_{\mathrm{s}}^{2} \Sigma_{\mathrm{d}}\left(V_{\mathrm{s}}\right)^{2} 2(1-\cos i)$

which differs from $\Delta E$ by $1 / \cos i$; we will denote $2(1-\cos i)=\phi_{1}(i)=\phi(i) / \cos i$. 
Hereafter we will use the Solar units $r_{\mathrm{s}}=R_{\mathrm{s}} / R_{\odot}$; $m_{\mathrm{s}}=M_{\mathrm{s}} / M_{\odot} ;$ so, the cross section of a star is $\pi R_{\mathrm{s}}^{2} \simeq$ $1.54 \times 10^{22} r_{\mathrm{s}}^{2} \mathrm{~cm}^{2}$. We also define the usual values $m_{8}=$ $M_{\mathrm{BH}} / 10^{8} M_{\odot}, \dot{m}=(\mathrm{d} M / \mathrm{d} t) / \dot{M}_{\mathrm{Ed}}, \dot{M}_{\mathrm{Ed}} \simeq m_{8} M_{\odot} \mathrm{yr}^{-1}$.

Supposing that the stellar orbits are close to circular, we can approximate the time interval between two crossings of the disk by a star as $\Delta T=\pi R / V_{\mathrm{s}} \simeq 1.4 \times$ $10^{8} m_{8}^{-1 / 2} r_{-2}^{3 / 2} \mathrm{~s}$.

Denoting $\Sigma_{\mathrm{d}}=10^{4} \Sigma_{4} \mathrm{~g} \mathrm{~cm}^{-2}$, one can define the energy dissipated per crossing

$\Delta E \simeq 0.7 \times 10^{44} Q r_{\mathrm{s}}^{2} \Sigma_{4} m_{8} r_{-2}^{-1} \phi_{1}(i) \mathrm{erg}$,

and the averaged rate of the energy change of a star, $\Delta E / \Delta T$ is

$\mathrm{d} E / \mathrm{d} t \simeq 0.5 \times 10^{36} Q r_{\mathrm{s}}^{2} \Sigma_{4} m_{8}^{3 / 2} r_{-2}^{-5 / 2} \phi(i) \mathrm{erg} \mathrm{s}^{-1}$.

The commonly accepted models for the AD are so-called $\alpha$-disks, the Shakura-Sunyaev model (Shakura \& Sunyaev 1973, hereafter SS), and the Novikov \& Thorne (1973) model (hereafter NT); the last was used in Rauch (1995). On the other hand, physical assumptions of the models, the geometrically thin and optically thick of the $\alpha$-disks theory, are not universal. Other processes like energy advection, temperature instability, self-gravity and the CSC gravity are essential in different cases (see the recent review of Park \& Ostriker 2001).

We will use mostly the NT model, as it contains a simple radial dependence of the surface density of the $\mathrm{AD}$; according to Rauch (1995) the surface density of the AD is

$\Sigma_{4} \simeq 2.7 \times 10^{2} m_{8}^{1 / 4} \alpha_{-2}^{-4 / 5}\left(\dot{m}_{-1}\right)^{7 / 10} r_{-2}^{-3 / 4}$.

Then the energy dissipation per crossing is

$\Delta E \simeq 1.9 \times 10^{46} Q r_{\mathrm{s}}^{2} m_{8}^{5 / 4} \alpha_{-2}^{-4 / 5} \dot{m}_{-1}^{7 / 10} r_{-2}^{-7 / 4} \phi_{1}(i) \mathrm{erg},(8)$

and the average rate of the energy change of a star is

$\mathrm{d} E / \mathrm{d} t \simeq 1.35 \times 10^{38} Q r_{\mathrm{s}}^{2} m_{8}^{7 / 4} r_{-2}^{-13 / 4} \phi(i) \mathrm{erg} \mathrm{s}^{-1}$.

According to the SS model, the parameters of the accretion disk behave differently in three regions $(\mathrm{a}, \mathrm{b}$, c), and we have from SS the positions of $a / b$ and $b / c$ borders: $r_{\mathrm{ab}-2}=2.6 m_{8}^{23 / 21} \alpha^{2 / 21}\left(\dot{m} \varphi_{\mathrm{ab}}\right)^{16 / 21} ; r_{\mathrm{bc}-2}=$ $18.9 m_{8}\left(\dot{m} \varphi_{\mathrm{bc}}\right)^{2 / 3}$; where $r_{\mathrm{ab}-2}=R_{\mathrm{ab}} / 10^{-2} \mathrm{pc}, \varphi=$ $1-r^{-1 / 2}$.

The surface density and the height scale in the three zones may be written as

$$
\text { a) } \begin{aligned}
\Sigma & \simeq 2.8 \times 10^{4}(\alpha \dot{m} \varphi)^{-1} m_{8}^{-3 / 2} r_{-2}^{3 / 2} \mathrm{~g} \mathrm{~cm}^{-2}, \\
z & \simeq 3.2 \times 10^{14} \cdot m m_{8} \varphi \mathrm{cm}, \\
\text { b) } \Sigma & \simeq 2.1 \times 10^{5} \alpha^{-4 / 5} m_{8}^{4 / 5}(\dot{m} \varphi)^{3 / 5} r_{-2}^{-3 / 5}, \\
z & \simeq 0.85 \times 10^{14} \alpha^{-1 / 10} m_{8}^{39 / 20}(\dot{m})^{2 / 5} \varphi^{1 / 5} r_{-2}^{21 / 20}, \\
\text { c) } \Sigma & \simeq 3.11 \times 10^{5} \alpha^{-4 / 5}(\dot{m} \varphi)^{7 / 10} m_{8}^{19 / 20} r_{2}^{-3 / 4}, \\
z & \simeq 0.67 \times 10^{14} \alpha^{-1 / 5} m_{8}^{-1 / 10}(\dot{m} \varphi)^{3 / 20} r_{-2}^{9 / 8} .
\end{aligned}
$$

With these relations, the energy dissipation per crossing in the $\mathrm{a}, \mathrm{b}$,and $\mathrm{c}$ zones of the disk are (in $10^{45} \mathrm{erg}$ ):
a) $\Delta E \simeq 0.2 Q r_{\mathrm{s}}^{2}(\alpha \dot{m})^{-1} m_{8}^{-1 / 2} r_{-2}^{1 / 2} \phi_{1}(i)$,
b) $\Delta E \simeq 1.47 Q r_{\mathrm{s}}^{2} \alpha^{-4 / 5}(\dot{m})^{3 / 5} m_{8}^{9 / 5} r_{-2}^{-8 / 5} \phi_{1}(i)$
c) $\Delta E \simeq 2.18 Q r_{\mathrm{s}}^{2} \alpha^{-4 / 5}(\dot{m})^{7 / 10} m_{8}^{39 / 20} r_{-2}^{-7 / 4} \phi_{1}(i)$.

The average energy losses of a star for the three zones are (in $10^{37} \mathrm{erg} \mathrm{s}^{-1}$ ):
a) $\mathrm{d} E / \mathrm{d} t=0.14 Q r_{\mathrm{s}}^{2}(\alpha \dot{m})^{-1} r_{-2}^{-1} \phi(i)$
b) $\mathrm{d} E / \mathrm{d} t=1.1 Q r_{\mathrm{s}}^{2} \alpha^{-4 / 5}(\dot{m})^{3 / 5} m_{8}^{23 / 10} r_{-2}^{-21 / 10} \phi(i)$
c) $\mathrm{d} E / \mathrm{d} t=1.6 Q r_{\mathrm{s}}^{2} \alpha^{-4 / 5}(\dot{m})^{7 / 10} m_{8}^{49 / 20} r_{-2}^{-3 / 4} \phi(i)$.

\subsection{The star-star interactions and the inflow of stars}

In spherically symmetrical conditions, the influence of the central massive black hole on the stellar cluster structure and the inflow of stars into the $\mathrm{MBH}$ was considered by Peebles (1972) and solved by Bahcall \& Wolf (1976) (hereafter BW). BW showed that the presence of a $\mathrm{MBH}$ in a dense stellar cluster typically leads to the creation of a central stellar cusp with a density profile $n(R)=n_{0}\left(R / R_{\mathrm{a}}\right)^{q}$, with $q \simeq-7 / 4$ and $R_{\mathrm{a}} \simeq R_{\mathrm{ss}} M_{\mathrm{BH}} / M_{\mathrm{SS}}$. The stellar distribution function depends on the stellar energy as $f(E) \sim E^{1 / 4}$, where $E=G M_{\mathrm{BH}} / r-1 / 2 V_{\mathrm{s}}^{2}$ and $V_{\mathrm{s}}$ is the stellar velocity. Both the creation of the cusp and the inflow of the stars into the MBH are due to star-star gravitational interaction. The result was obtained for an isotropic stellar distribution function, but BW showed that it is valid if we use anisotropic distribution functions as well. However, in the case of AGNs with accretion disks, the interactions of the stars with the disk in the central part of the stellar system are inevitable, and the influence of the interaction on the stellar dynamics have to be taken into account.

Let us compare the rate of the energy changes of a typical star (let it be the solar-type star) for the cases of star-star (hereafter s-s) interaction and star-disk interaction. For the former case one has

$\mathrm{d} E^{(\mathrm{ss})} / \mathrm{d} t \simeq 4 \pi G^{2} M_{\mathrm{s}}^{3} V_{\mathrm{sr}}^{-1} n_{\mathrm{s}} \ln \Lambda$,

where $V_{\text {sr }}$ is the effective relative velocity of the stars, $n_{\mathrm{s}}$ is the stellar density and $\ln \Lambda$ is the Coulomb logarithm; for the latter case Eq. (6) applies.

It is essential to note that in the present work we will use some effective values of physical variables. In principle these variables show a certain distribution at each radius: stars at each radius have various inclinations, velocities relative to the disk etc. A complex numerical approach would be necessary in order to model the distributions of these quantities. Knowing the distributions we would be able to compute accurately the mean or median values of the parameters. However, such a program is far beyond the scope of the present paper. Instead, we reduce the dimension of the problem by introducing a priori some effective values at each radius, with values following from 
processes we consider. It permits us to determine physically reasonable radial trends; but it is obvious that the obtained results are true only in a qualitative sense.

If the mass of the CSC is larger than the mass of MBH, the effective velocity of the stars in the outer parts of the CSC is close to the virial velocity $V_{\mathrm{s}} \approx\left(G M_{\mathrm{sc}} / R_{\mathrm{sc}}\right)^{1 / 2}$, where $M_{\mathrm{sc}}$ and $R_{\mathrm{sc}}$ are the mass and radius of the CSC. In the inner part of the $\mathrm{AD}$ the velocity at the almost circular orbits is close to $V_{\mathrm{s}} \approx(G M / R)^{1 / 2}$, where $M(R)=$ $M_{\mathrm{BH}}+M_{\mathrm{sc}}(R)+M_{\mathrm{AD}}(R)$ is the total mass within the radius $R$.

Rauch (1995) has shown that close to a black hole the characteristic two-body relaxation time $t_{\mathrm{ss}} \sim$ $v^{3} /\left(\pi G^{2} M_{\mathrm{s}}^{2} n_{\mathrm{s}}\right) \sim 10^{12}\left(n_{\mathrm{s}} / 10^{7} \mathrm{pc}^{-3}\right)$ is much longer than the characteristic time of the stellar orbit alignment with the disk, $t_{\mathrm{i}} \sim t_{\text {orb }} M_{\mathrm{s}} /\left(\pi R_{\mathrm{s}}^{2}\right) / \Sigma_{\mathrm{d}}$. This is still true in our case of more dense CSC if it is spherically symmetric, which can be seen from the numerical estimates of the correspondent energy-loss rates (in $\mathrm{erg} \mathrm{s}^{-1}$ ):

$\mathrm{d} E^{(\mathrm{ss})} / \mathrm{d} t \simeq 5.9 \times 10^{32} m_{\mathrm{s}}^{3} m_{8}^{-1 / 2} r_{-2}^{1 / 2} n_{10}(\ln \Lambda)_{1}$,

$\mathrm{d} E^{(\mathrm{sd})} / \mathrm{d} t \simeq 5 \times 10^{35} Q \Sigma_{4} r_{\mathrm{s}}^{2} m_{8}^{3 / 2} r_{-2}^{-5 / 2}$,

where $m_{8}=M(R) / 10^{8} M_{\odot}, n_{10}=n_{\mathrm{s}} /\left(10^{10} \mathrm{pc}^{-3}\right)$.

One can see that the star-disk interaction is relatively strong even in the outer parts of the AD. We can estimate the critical outer radius of the region where the two interactions became comparable, if the inclination of the orbits is negligible $\left[\sin \left(i_{\mathrm{ef}}\right) \sim 1\right]$ in this outer region:

$r_{-2}^{\text {crit }} \sim 9.5 \Sigma_{4}^{1 / 3} r_{\mathrm{s}}^{2 / 3} m_{\mathrm{s}}^{-1} m_{8}^{2 / 3}\left[n_{10}(\ln \Lambda)_{1}\right]^{-1 / 3}$,

that is $R^{\text {crit }} \simeq 0.1 \mathrm{pc}$ (if $\Sigma_{4}=m_{8}=n_{10}=1$ ).

At smaller radii the inelastic star-disk collisions become important, and the stellar orbits incline to the disk. If the orbit evolution starts from a non-inclined orbit, the orbit will quickly decline to the disk plane (as was shown by Rauch 1995). But when the inclination angle becomes small, the situation can change due to the increased stellar density. The reason is that the inclination of the orbits influences the relative velocities, and consequently the relaxation times of the star-disk and star-star collisions.

We will assume that the inclination angles of the orbits are smoothly distributed above the disk, and the distribution can be characterized by some effective inclination angle $i_{\mathrm{ef}}$. The characteristic relative velocities of stars in the co-rotating (with the disk) coordinate system are then close to $V_{\mathrm{sr}} \simeq V_{\mathrm{s}} \sin i_{\mathrm{ef}}$, that is close to the average velocity component normal to the disk. Introducing the effective inclination angle into the expressions of the energy changes due to the star-star and star-disk interactions, we have from Eqs. (6) and (14) numerically (in $\mathrm{erg} \mathrm{s}^{-1}$ ):

$\mathrm{d} E^{(\mathrm{ss})} / \mathrm{d} t \simeq 5.9 \times 10^{32} m_{\mathrm{s}}^{3} m_{8}^{-1 / 2} r_{-2}^{1 / 2} n_{10}(\ln \Lambda)_{1} / \sin \left(i_{\mathrm{ef}}\right),(17)$

$\mathrm{d} E^{(\mathrm{sd})} / \mathrm{d} t \simeq 5 \times 10^{35} Q \Sigma_{4} r_{\mathrm{s}}^{2} m_{8}^{3 / 2} r_{-2}^{-5 / 2} \phi\left(i_{\mathrm{ef}}\right)$.

The important point is that the s-s interaction behaves as $1 / \sin \left(i_{\mathrm{ef}}\right)$ and the $\mathrm{s}-\mathrm{d}$ interaction is proportional to $\left[\sin \left(i_{\mathrm{ef}}\right)\right]^{2}$, so both interactions may be comparable at small angles. It is intuitively clear, that an equilibrium situation exists when $\mathrm{d} E^{(\mathrm{ss})} / \mathrm{d} t \simeq \mathrm{d} E^{(\mathrm{sd})} / \mathrm{d} t$. The process is similar to the Brownian motion of the normal velocity component $V \sin i$, and the equilibrium velocity dispersion corresponds to an equilibrium of braking and fluctuation forces in the Langevin equation.

As the characteristic time of approaching the equilibrium is relatively short (close to the characteristic time of orbit inclination, $\left.t_{\mathrm{i}} \simeq t_{\mathrm{dyn}}\left(M_{\mathrm{s}} / \pi R_{\mathrm{s}}^{2}\right) / \Sigma_{\mathrm{d}}\right)$, we will suppose further that the equilibrium condition $\mathrm{d} E^{(\mathrm{ss})} / \mathrm{d} t \simeq$ $\mathrm{d} E^{(\mathrm{sd})} / \mathrm{d} t$ is realized at any distance $R<R^{\text {crit }}$. This equilibrium means that in a dense enough CSC the stars will never be completely incorporated into the disk plane: when orbits are inclined too much, the pair stellar interactions will come into play, and they will scatter the stars to less inclined orbits (the eccentricities of the orbits will also be scattered to the same order of magnitude $e_{\mathrm{ef}} \sim \sin i$ ). The immediate consequence of this property, which leads to the fluctuation of the inclination angle around some effective value, is that the fluctuating drag force maintains some constant dissipative flow of the stars to the center of the disk. The rate of the inflow is

$V_{\mathrm{R}}=\mathrm{d} R / \mathrm{d} t=\mathrm{d} R / \mathrm{d} E(\mathrm{~d} E / \mathrm{d} t)$

where $\mathrm{d} E / \mathrm{d} R=G M_{\mathrm{BH}} M_{\mathrm{s}} / R^{2} \quad \mathrm{erg} \mathrm{cm}^{-1}$, that is $\mathrm{d} R / \mathrm{d} E \simeq 3.37 \times 10^{-35} m_{\mathrm{s}}^{-1} m_{8}^{-1} r_{-2}^{2} \mathrm{~cm} \mathrm{erg}^{-1}$, and $\mathrm{d} E / \mathrm{d} t$ is the averaged dissipative energy loss of the star. We therefore obtain

$V_{\mathrm{R}} \simeq 17 Q \Sigma_{4} r_{\mathrm{s}}^{2} m_{\mathrm{s}}^{-1} m_{8}^{1 / 2} r_{-2}^{-1 / 2} \phi(i) \mathrm{cm} \mathrm{s}^{-1}$.

In stationary conditions, the flow of the stars is

$\mathrm{d} n_{\mathrm{s}} / \mathrm{d} t=\dot{n} \simeq 4 \pi n_{\mathrm{s}} V_{\mathrm{R}} R^{2} \sin \left(i_{\mathrm{ef}}\right)$,

where $n_{\mathrm{s}}$ is the stellar density at the $\mathrm{AD}$ plane and $i_{\mathrm{ef}}(R)$ is the effective inclination angle of stellar orbits, which means also the part of the solid angle where the inflow is concentrated.

Numerically, this formula gives the flow:

$\dot{n}_{y} \simeq 2 \times 10^{-3} n_{10} Q \Sigma_{4} r_{\mathrm{s}}^{2} m_{\mathrm{s}}^{-1} m_{8}^{1 / 2} r_{-2}^{3 / 2} \sin i_{\mathrm{ef}} \phi\left(i_{\mathrm{ef}}\right) \mathrm{yr}^{-1}(22)$

in units of stars/year, where $n_{10}$ is the stellar density in units $10^{10} \mathrm{pc}^{-3}$.

\section{The distribution of stars in the central part of CSC}

\subsection{Constant stellar masses and inflow of the stars}

As was shown in the previous section, the stellar orbits tend to decline to the disk and to shrink due to the energy loss of the stars crossing the disk, but the inclination of the orbits is limited by the scattering due to the gravitational star-star interaction. In the stationary case both processes are in equilibrium, which defines the inflow of the stars to the center. 
This process can be described with a system of two equations, which permit us to find the distribution of the stars. The first equation is the star inflow Eq. (22), and the second is the condition of equilibrium of the inelastic (star-disk) and elastic (star-star) collisions,

$\mathrm{d} E^{(\mathrm{sd})} / \mathrm{d} t=\mathrm{d} E^{(\mathrm{ss})} / \mathrm{d} t$.

Equation (22) can be written in the form

$\sin \left(i_{\mathrm{ef}}\right) \phi\left(i_{\mathrm{ef}}\right) n_{10} \simeq 5 \times 10^{2} \dot{n}_{\mathrm{y}} /\left(Q \Sigma_{4}\right) m_{\mathrm{s}} r_{\mathrm{s}}^{-2} m_{8}^{-1 / 2} r_{-2}^{-3 / 2}$

where $\dot{n}_{y}$ is the stellar inflow in the units $1 /$ year, $n_{10}$ is the stellar density in $10^{10} \mathrm{pc}^{-3}$. On the other hand, from Eq. (23), using Eqs. (17) and (18) one obtains:

$$
\begin{aligned}
& \sin \left(i_{\mathrm{ef}}\right) \phi\left(i_{\mathrm{ef}}\right) / n_{10} \simeq \\
& 1.2 \times 10^{-3}\left(Q \Sigma_{4}\right)^{-1} m_{\mathrm{s}}^{3} r_{\mathrm{s}}^{-2} m_{8}^{-2} r_{-2}^{3}(\ln \Lambda)_{1} .
\end{aligned}
$$

Equations (24) and (25) form a system of equations for two variables: $n_{10}(r)$ and $i_{\mathrm{ef}}(r)$. Dividing Eq. (24) by (25) one has

$n_{10}(r) \simeq 0.6 \times 10^{3}\left[\dot{n}_{y} /(\ln \Lambda)_{1}\right]^{1 / 2} m_{\mathrm{s}}{ }^{-1} m_{8}^{3 / 4} r_{-2}^{-9 / 4}$.

This distribution is independent of the disk parameters, paradoxically at first glance. The reason is the underlying assumption of the equilibrium of non-elastic (s-d) and elastic (s-s) processes, but this condition is satisfied only in dense enough disks, so it is not valid for low surface density disks.

Multiplying Eq. (24) by (25), and using (for $i_{\mathrm{ef}}<\pi / 3$ ) the approximation $\phi(i)=2 \sin ^{2} i \cos i /(1+\cos i) \approx \sin ^{2} i$, one has

$$
\begin{aligned}
& \sin \left(i_{\mathrm{ef}}\right) \approx \\
& \quad 0.9\left[\dot{n}_{y}(\ln \Lambda)_{1}\right]^{1 / 6}\left(Q \Sigma_{4}\right)^{-1 / 3}\left(m_{\mathrm{s}} / r_{\mathrm{s}}\right)^{2 / 3} m_{8}^{-5 / 12} r_{-2}^{1 / 4} .
\end{aligned}
$$

This solution is valid in the innermost parts of the CSC/AD system, where $\sin \left(i_{\mathrm{ef}}\right)<1$; in the outermost parts the orbits are non-circular and we pose $\sin \left(i_{\mathrm{ef}}\right)=1$ there.

If we use the disk surface density $\Sigma$ of the NT disk from Rauch (1995), then

$$
\begin{aligned}
\sin \left(i_{\mathrm{ef}}\right) \approx & 0.14\left[\dot{n}_{y}(\ln \Lambda)_{1}\right]^{1 / 6}(Q)^{-1 / 3} \\
& \times\left(m_{\mathrm{s}} / r_{\mathrm{s}}\right)^{2 / 3} m_{8}^{-1 / 2} \alpha_{-2}^{4 / 15}\left(\dot{m}_{-1}\right)^{-7 / 30} r_{-2}^{1 / 2} .
\end{aligned}
$$

The dependences of $\sin i_{\mathrm{ef}}$ on $r$ for three zones of the SS accretion disk are:

$\sin \left(i_{\text {ef }}\right)(r) \simeq$

a) $0.64 Q^{-1 / 3}\left(m_{\mathrm{s}} / r_{\mathrm{s}}\right)^{2 / 3}(\alpha \dot{m})^{1 / 3} m_{8}^{1 / 12}\left[\dot{n}_{\mathrm{y}}(\ln \Lambda)_{1}\right]^{1 / 6} r_{-2}^{-1 / 4}$

b) $0.34 Q^{-1 / 3}\left(m_{\mathrm{s}} / r_{\mathrm{s}}\right)^{2 / 3}(\alpha)^{4 / 15}(\dot{m})^{-1 / 5} m_{8}^{-41 / 60}$

$$
\times\left[\dot{n}_{\mathrm{y}}(\ln \Lambda)_{1}\right]^{1 / 6} r_{-2}^{9 / 20}
$$

c) $0.3 Q^{-1 / 3}\left(m_{\mathrm{s}} / r_{\mathrm{s}}\right)^{2 / 3}(\alpha)^{4 / 15}(\dot{m})^{-7 / 30} m_{8}^{-11 / 15}$

$$
\times\left[\dot{n}_{\mathrm{y}}(\ln \Lambda)_{1}\right]^{1 / 6} r_{-2}^{1 / 2} \text {. }
$$

The dependence of $\sin \left(i_{\text {ef }}\right)$ and $n_{10}$ on $r_{-2}$ for different $\Sigma_{4}(r)$ (the SS, NT and constant density disk cases) are

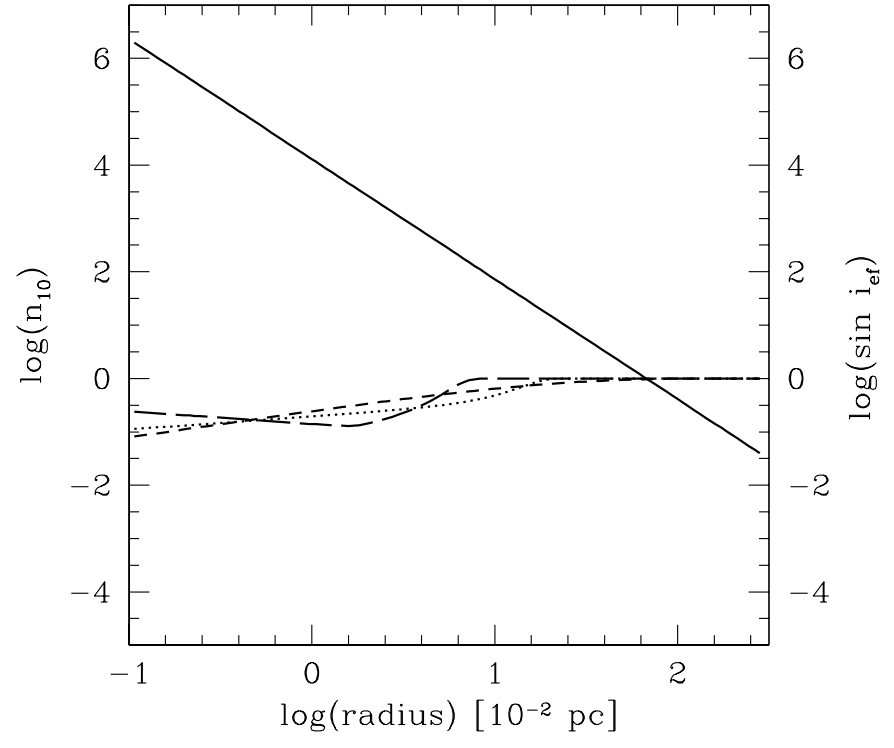

Fig. 1. Radial dependence of stellar density $n_{10}$ (continuous line) and $\sin \left(i_{\mathrm{ef}}\right)$ in the case of constant stellar flow for SS disk, constant surface density disk and NT disk (longdashed, short dashed and dotted line, correspondingly).

shown in Fig. 1 . We adopt $M_{\mathrm{BH}}=10^{8} M_{\odot}, \alpha=0.01$ and $\dot{m}=1$. One can see that the stellar density rises quickly at small radii, while the effective inclination angle of the stellar orbits diminishes to the center. As can be seen from the picture, the difference in the behavior of $\sin \left(i_{\mathrm{ef}}\right)$ for different disk models is not too large (though it has a wide minimum in the case of an SS disk), so further on we will use the NT model for our model calculations.

The inflow rate of the stars $\dot{n}_{\mathrm{y}}$ in this consideration (in the approximate model we investigate here) is assumed to be given by a constant, which is determined by the conditions at the outer border, $R=R_{\text {out }}$. This outer border is close to the above-estimated value $R^{\text {crit }}$, determined by condition (16). Physically, the value $\dot{n}\left(R_{\text {out }}\right)$ is determined by the diffusion process, which depends on the properties of the compact stellar cluster, and which for the case of a non-rotating CSC was described by Bahcall \& Wolf (1976). It is clear that in the central part of the CSC the stellar density can be so large that physical collisions of the stars can occur; besides, the stars can lose mass when they cross the disk. These processes have to be taken into account.

\subsection{The contact stellar collisions}

Let us consider the change of stellar flow due to the contact (sometimes it is called "physical") collisions of the stars; this is the non-elastic s-s process. The collision velocities are about $V_{\mathrm{c}} \sim V \sin i$ and the escape velocity from the surface of a star can be estimated as $v_{*} \simeq 6 \times 10^{7}\left[m_{\mathrm{s}} / r_{\mathrm{s}}\right]^{1 / 2} \mathrm{~cm} \mathrm{~s}^{-1}$. If $V_{\mathrm{c}}>v_{*}$, the averaged mass loss per collision is large (Murphy et al. 1991), and stars are completely disrupted if the impact parameter is 
less than some value. In the opposite case $V_{\mathrm{c}}<v_{*}$ (if, say, $\left.\sin \left(i_{\mathrm{ef}}\right) \ll 1\right)$, the collisions can produce binary stars, or stars can be merged. Within the framework of the present paper we adopt equal masses of stars (which itself is far enough from reality!). In contact collisions a fraction of mass is always lost, but merging can increase stellar mass. Therefore, we will assume that both processes somehow compensate, and consider unchanged stellar masses, but take into account a decrease of the stellar density $n_{\mathrm{s}}$ and the stellar inflow $\dot{n}_{\mathrm{y}}$ as a result of the direct s-s collisions.

In Sect. 3.1 we have obtained formulae for the stellar density $n_{\mathrm{s}}$ and $\sin \left(i_{\mathrm{ef}}\right)$, depending on the inflow of stars $\dot{n}_{y}$. These formulae are valid also for the case $\dot{n}_{y}=\dot{n}_{y}(R)$. The frequency of the physical collisions of a given star with another stars (all stars have stellar radii $R_{\mathrm{s}}$ ) is

$\nu_{\mathrm{Ss}}^{1}=4 \pi n_{\mathrm{s}} R_{\mathrm{s}}^{2} V_{\mathrm{s}} \sin \left(i_{\mathrm{ef}}\right)$,

and numerically:

$$
\begin{aligned}
\nu_{\mathrm{Ss}}^{1} \simeq & 0.4 \times 10^{-12}\left[\dot{n}_{y}(\ln \Lambda)_{1}\right]^{1 / 2} \\
& \times m_{\mathrm{s}}^{-1} r_{\mathrm{s}}^{2} m_{8}^{5 / 4} r_{-2}^{-11 / 4} \sin \left(i_{\mathrm{ef}}\right) \mathrm{s}^{-1} .
\end{aligned}
$$

The frequency of the star-star physical (contact) collisions in the spherical layer is

$\mathrm{d} \nu_{\mathrm{ss}}=16 \pi^{2} R_{\mathrm{s}}^{2} n_{\mathrm{s}}^{2} V_{\mathrm{sr}}^{2}\left[\sin \left(i_{\mathrm{ef}}\right)\right]^{2} \mathrm{~d} R$,

thus giving,

$$
\begin{aligned}
\mathrm{d} \nu_{\mathrm{ss}} \simeq & 0.95 \times 10^{-4} \\
& \times r_{\mathrm{s}}^{2} m_{\mathrm{s}}^{-2} m_{8}^{2} \dot{n}_{y}(\ln \Lambda)_{1}^{-1} r_{-2}^{-3} \sin ^{2}\left(i_{\mathrm{ef}}\right) \mathrm{d} r_{-2} \mathrm{~s}^{-1}
\end{aligned}
$$

The rate of the diminishing of the stellar inflow due to disruption of stars is $\mathrm{d}\left(\dot{n}_{y}\right) / \mathrm{d} r=-q_{\mathrm{v}} \mathrm{d} \nu_{\mathrm{ss}}(1 / \mathrm{s})\left(3 \times 10^{7} \mathrm{~s} / \mathrm{yr}\right)$, where $q_{\mathrm{v}}$ is equal to 2 or 1 , for the disruptive or merging case, depending on the $V_{\mathrm{r}} / v_{*}$ relation. Besides, the cross-section of the stellar collisions depends on the relative stellar velocity $V_{\mathrm{r}} / v_{*}$ due to gravitational focusing at small velocities. We use these dependences in the numerical calculations (see below). Here we will neglect them and obtain approximate solutions with $q_{\mathrm{v}}=1$.

In the spherical layer between $R$ and $R+\mathrm{d} R$

$\mathrm{d}\left(\dot{n}_{y}\right) \simeq$

$$
2.9 \times 10^{3} \dot{n}_{y}(\ln \Lambda)_{1}^{-1} m_{\mathrm{s}}{ }^{-2} r_{\mathrm{s}}^{2} m_{8}^{2} \sin ^{2}\left(i_{\mathrm{ef}}\right) r_{-2}^{-3} \mathrm{~d} r_{-2} .
$$

Using $\sin \left(i_{\mathrm{ef}}\right)$ from Eq. $(27)$, one has $\left(\dot{n}_{y}\right.$ in units $\left.\mathrm{yr}^{-1}\right)$

$$
\begin{aligned}
& \mathrm{d}\left(\dot{n}_{y}\right) \simeq 1.25 \times 10^{3} \dot{n}_{y}^{4 / 3} \\
& \quad \times\left(Q \Sigma_{4}\right)^{-2 / 3}(\ln \Lambda)_{1}^{-2 / 3} m_{\mathrm{s}}{ }^{-2 / 3} r_{\mathrm{s}}^{2 / 3} m_{8}^{7 / 6} r_{-2}^{-5 / 2} \mathrm{~d} r_{-2} .
\end{aligned}
$$

The solution of the differential equation can be obtained for different $\Sigma(r)=A r^{u}$. If we assume $\Sigma=$ const., the solution is

$$
\begin{aligned}
& \dot{n}_{y}(R) \simeq\left\{\dot{n}_{0 y}^{-1 / 3}+0.26 \times 10^{3}\left(Q \Sigma_{4}\right)^{-2 / 3}\right. \\
& \left.\quad \times(\ln \Lambda)_{1}^{-2 / 3} m_{\mathrm{s}}{ }^{-2 / 3} r_{\mathrm{s}}^{2 / 3} m_{8}^{7 / 6}\left[r_{-2}^{-3 / 2}-r_{0-2}^{-3 / 2}\right]\right\}^{-3} .
\end{aligned}
$$

If we use $\Sigma$ from the NT model, then

$$
\begin{gathered}
\mathrm{d}\left[\dot{n}_{y}(R)\right] \simeq 0.5 \times 10^{2}\left[\dot{n}_{y}(R)\right]^{4 / 3}(Q)^{-2 / 3}(\ln \Lambda)_{1}^{-2 / 3} \\
\quad \times m_{\mathrm{s}}^{-2 / 3} r_{\mathrm{s}}^{2 / 3} m_{8}^{5 / 6} \alpha_{-2}^{8 / 15}\left(\dot{m}_{-1}\right)^{-7 / 15} r_{-2}^{-2} \mathrm{~d} r_{-2},
\end{gathered}
$$

and the approximate solution is

$$
\begin{aligned}
& \dot{n}_{y}(R) \simeq\left\{\dot{n}_{0 y}^{-1 / 3}+3.4(Q)^{-2 / 3}(\ln \Lambda)_{1}^{-2 / 3}\right. \\
& \left.\quad \times m_{\mathrm{s}}{ }^{-2 / 3} r_{\mathrm{s}}^{2 / 3} m_{8}^{5 / 6} \alpha_{-2}^{8 / 15}(\dot{m})^{-7 / 15}\left[r_{-2}^{-1}-r_{0-2}^{-1}\right]\right\}^{-3} .
\end{aligned}
$$

With these results and using Eqs. (26) and (27), we have the stellar density and the effective inclination angle of orbits

$$
\begin{aligned}
n_{10}(R) \approx & 0.67 \times 10^{3}\left[\dot{n}_{y}(R) /(\ln \Lambda)_{1}\right]^{1 / 2} m_{8}^{3 / 4} r_{-2}^{-9 / 4} m_{\mathrm{s}}^{-1} \\
\sin \left(i_{\mathrm{ef}}\right)(R) & \approx 0.93\left[\dot{n}_{y}(R)(\ln \Lambda)_{1}\right]^{1 / 6} \\
\times & \left(Q \Sigma_{4}\right)^{-1 / 3}\left(m_{\mathrm{s}} / r_{\mathrm{s}}\right)^{2 / 3} m_{8}^{-5 / 12} r_{-2}^{1 / 4}
\end{aligned}
$$

A more precise result can be obtained numerically if we take into account the dependence of the cross-section of the stellar collisions on the relative stellar velocity due to gravitational focusing at small velocities $V_{\text {rel }} \leq V_{\text {esc }} \simeq$ $6 \times 10^{7}\left(m_{\mathrm{s}} / r_{\mathrm{s}}\right)^{1 / 2} \mathrm{~cm} \mathrm{~s}^{-1}$. These cases take place at small $\sin i_{\text {ef }}$ and/or at large distances, where we have to use collision cross sections as $\sigma_{\mathrm{ss}}=4 \pi R_{\mathrm{s}}^{2}\left(1+V_{\mathrm{esc}} / V_{\mathrm{rel}}\right)^{2}$.

\subsection{Stellar mass loss due to s-d interactions}

In the above considerations we neglected the change of the masses of stars due to s-s collisions, assuming that mass loss and stellar merging compensate and that stellar masses keep their initial values. This approximation is reasonable at large radii since the stellar density is small, and collisions of stars are rare. On the other hand, stars would systematically lose mass when crossing the disk, and this process is more intense at small radii.

The frequency of the star-disk interactions of a single star in a circular orbit with radius $R$ is inversely proportional to the half-period, $\nu_{\mathrm{sd}}=1 / \Delta T(R)$; numerically

$\nu_{\mathrm{sd}}^{1} \simeq 0.71 \times 10^{-8} m_{8}^{1 / 2} r_{-2}^{-3 / 2} \mathrm{~s}^{-1}$.

The differential frequency of the star-disk crosses by stars from the volume of a spherical layer $\mathrm{d} V=$ $4 \pi R^{2} \sin \left(i_{\mathrm{ef}}\right) \mathrm{d} R$ is

$\mathrm{d} \nu_{\mathrm{sd}}=4 \pi n_{\mathrm{s}} \Delta T^{-1} R^{2} \sin \left(i_{\mathrm{ef}}\right) \mathrm{d} R$,

and expressed in $\left[\mathrm{s}^{-1}\right]$ gives

$\mathrm{d} \nu_{\mathrm{sd}} \simeq 0.6 m_{\mathrm{s}}^{-1} m_{8}^{5 / 4}\left[\dot{n}_{y} /(\ln \Lambda)_{1}\right]^{1 / 2} r_{-2}^{-7 / 4} \sin \left(i_{\mathrm{ef}}\right) \mathrm{d} r_{-2}$

As the effective inclination angle is non-zero (and typically it is larger then several degrees), the star-disk velocities are supersonic and the stars lose their masses when crossing the disk. Let us suppose that the mass-loss rate is proportional to the dissipated energy

$\mathrm{d} M_{\mathrm{s}} / \mathrm{d} t=q_{\mathrm{m}}(\mathrm{d} E 1 / \mathrm{d} t)(\mathrm{d} M / \mathrm{d} E)$

where $\mathrm{d} E 1 / \mathrm{d} t$ is the rate of the energy dissipation in the disk and $\mathrm{d} M_{\mathrm{s}} / \mathrm{d} E=-R_{\mathrm{s}} /\left(G M_{\mathrm{s}}\right)$.

Obviously, the envelopes of the red giants will be blown off in the process of crossing the outermost parts of a typical accretion disk of the bright AGN, so further on 


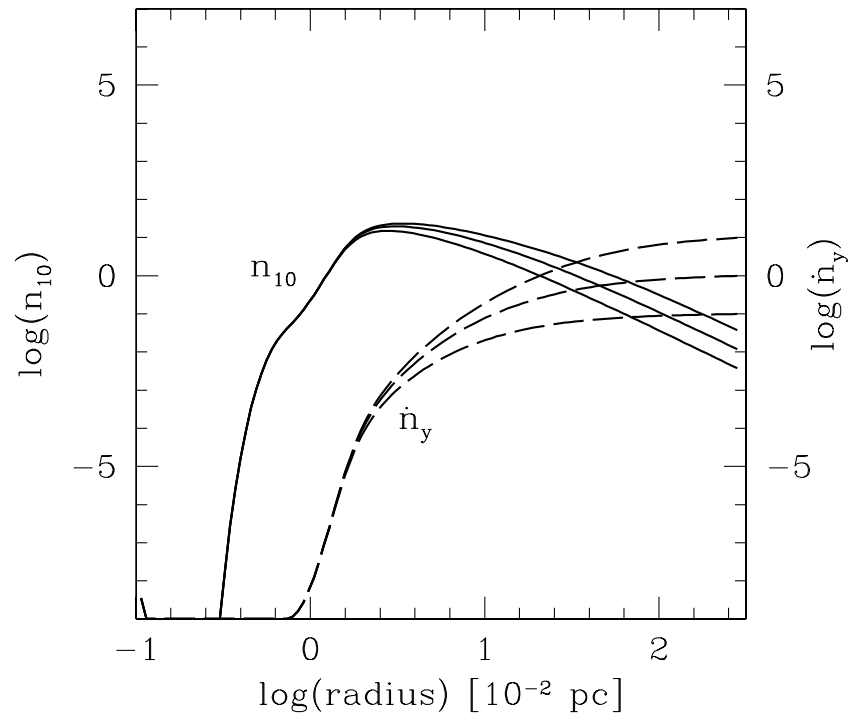

Fig. 2. The radial dependence of the stellar density $n_{10}$, and stellar flow $\dot{n}_{y}$ in the case of $M_{\mathrm{BH}}=10^{8} M_{\odot}$ and the values of the initial stellar flow equal $0.1,1.0$ and 10 stars/yr (dashed lines).

we will consider only the main-sequence stars. Denoting $q_{\mathrm{m}} \sim 10^{-2} q_{\mathrm{m}-2}$, and using Eqs. (4) and (6) we have (in units of $\mathrm{g} \mathrm{s}^{-1}$ )

$\mathrm{d} M_{\mathrm{s}} / \mathrm{d} t=-2.6 \times 10^{18} q_{\mathrm{m}-2} r_{\mathrm{s}}^{3} m_{\mathrm{s}}^{-1} Q \Sigma_{4} m_{8}^{3 / 2} r_{-2}^{-5 / 2} \phi_{1}\left(i_{\mathrm{ef}}\right)$.

We can deduce the radial dependence of $m_{\mathrm{s}}$ from $\mathrm{d} M_{\mathrm{s}} / \mathrm{d} R=\left(\mathrm{d} M_{\mathrm{s}} / \mathrm{d} t\right) / V_{\mathrm{r}}$. Assuming $\cos i_{\mathrm{ef}} \simeq 1$ and $r_{\mathrm{s}} \simeq$ $m_{\mathrm{s}}$ (appropriate for the main-sequence stars), one has

$\mathrm{d} m_{\mathrm{s}} / m_{\mathrm{s}} \simeq-2.3 q_{\mathrm{m}-2} m_{8}\left(\mathrm{~d} r_{-2} / r_{-2}^{2}\right)$.

The solution of the equation is

$m_{\mathrm{s}} \simeq m_{\mathrm{s} 0} \exp \left\{-K\left[1 / r_{-2}-1 / r_{-2}^{(0)}\right]\right\}$.

where $K=2.3 q_{\mathrm{m}-2} m_{8}$, and $r_{-2}^{(0)}=r_{-2}^{\text {crit }}$ is the outer (critical) radius of the stellar inflow. The peculiarity of the process is that (within our approximations!) it does not depend on the $\Sigma_{4}(r)$ behavior.

In the region $r_{-2} \ll r_{-2}^{(0)}$ the solution is $m_{\mathrm{s}} \simeq$ $m_{\mathrm{s} 0} \exp \left\{-K / r_{-2}\right\}$, and one can see that the mass of a star quickly goes to zero when $r_{-2}<K$. Physically this means that at this distance the time scale of the mass loss becomes less than that of the radial drift (inflow), so the stars with diminished masses crowd close to $r_{-2} \sim K$. The distance $R_{\mathrm{m}} \sim 0.01 K q_{\mathrm{m}-2}$ pc is the radius of melting of the stars due to the star-disk crossings.

It can be noted that the time between consecutive crossings is several years at this distance $\left(m_{8}=1\right)$, and the duration of crossing is about one day, so the stars really can be bloated due to deformation and heating of the surface, and this leads to even quicker melting of the stars.

Adopting a minimal stellar mass of $\sim 0.01 M_{\odot}$, one has $\left(r_{-2}\right)_{\min } \sim-K / \ln \left(m_{\mathrm{s} 0} / m_{\mathrm{s}}\right)$, that is $\left(r_{-2}\right)_{\min } \sim$ $0.5 q_{m-2} m_{8}$ for $m_{\mathrm{s} 0}=1$, and $\left(r_{-2}\right)_{\min } \sim q_{\mathrm{m}-2} m_{8}$ for $m_{\mathrm{s} 0}=0.1$.
As the stellar density drops quickly due to the melting of stars at $r_{-2}<\left(r_{-2}\right)_{\min }$, in the numerical calculation we used the approximation

$$
\begin{aligned}
n_{10} \simeq & 1.3 \times 10^{3}\left[\dot{n}_{y} /(\ln \Lambda)_{1}\right]^{1 / 2} \\
& \times m_{8}^{3 / 4} r_{-2}^{-9 / 4} m_{\mathrm{s} 0}^{-1} \exp \left\{\left[K / r_{-2}\left(1-0.5 K / r_{-2}\right]\right\},\right.
\end{aligned}
$$

that is the stellar density is enhanced close to $r_{-2} \approx$ $2.3 q_{m-2} m_{8}$ and then drops at smaller radii. This means the appearance of some "stellar hoop" with the radius $r_{-2}=2.3 q_{\mathrm{m}-2} m_{8}$. The numerical calculations of the emission line profiles (see Sect. 4) give reasonable results with $q_{\mathrm{m}-2} \simeq 1$, which we accept below as the standard value.

The influence of the process of star melting at small radii was taken into account in the numerical calculations of the models, and the results are presented in Figs. 2 and 3 .

Our approximation works for sufficiently dense disks, so we show the results of the numerical calculations for the case of NT disk with $\alpha=0.01$ and $\dot{m}=1$.

In Fig. 2 we show the radial dependences of stellar densities and stellar flows for the case of $M_{\mathrm{BH}}=10^{8} M_{\odot}$. with different initial (at the outer border) values of the stellar flow $\dot{n}_{y 0}=0.1,1,10 \mathrm{yr}^{-1}$. One can see that at small distances all the flows $\dot{n}_{y}(R)$ become almost the same and independent on the initial values of $\dot{n}_{y 0}$ at large radius. This result is notable, as it shows that in fact the accretion disk itself can be built with the gas resulting from the s-s disruptive collisions in the dense enough CSC.

We used this hypothesis for calculation of the reference models of different AGNs, assuming that the accretion rate onto the $\mathrm{MBH}$ is always equal to the initial mass inflow by stars at the outer border of the CSC/AD system, $\dot{n}_{y} m_{\mathrm{s}}=m_{8} \dot{m}$. In Fig. 3 we show the radial dependences of stellar densities, inclination angles and stellar flows for the reference models with $M_{\mathrm{BH}}=10^{7} M_{\odot}, 10^{8} M_{\odot}, 10^{9} M_{\odot}$; also cases without contact stellar collisions and mass loss due to the disk crossing are shown for comparison (thin lines), and the behavior of the stellar inflow $\dot{n}_{y}(R)$ is marked (dashed lines).

\section{Broad emission line region}

There is still no commonly accepted physical idea about the nature of the broad emission line regions (BELR) of AGNs. The possible role of the accretion disk was stressed by Dumont \& Collin-Souffrin (1990) and it was subsequently considered in a number of papers (e.g. Collin \& Hure 2001). There were, however, also many attempts to involve stars (viz. excited, bloated or cometlike stars) in the explanation of problems of clouds, moving and emitting in BELRs (Edvards 1980; Shull 1983; Penston 1988; Scoville \& Norman 1988, 1995; Kazans 1989; $\dot{Z}$ urek et al. 1994). The most recent was the study by Alexander \& Netzer $(1994,1997)$, where the authors considered emission from the outer envelopes of excited stars and used a spherically symmetric model of the structure and dynamics of the CSC in the vicinity of the 

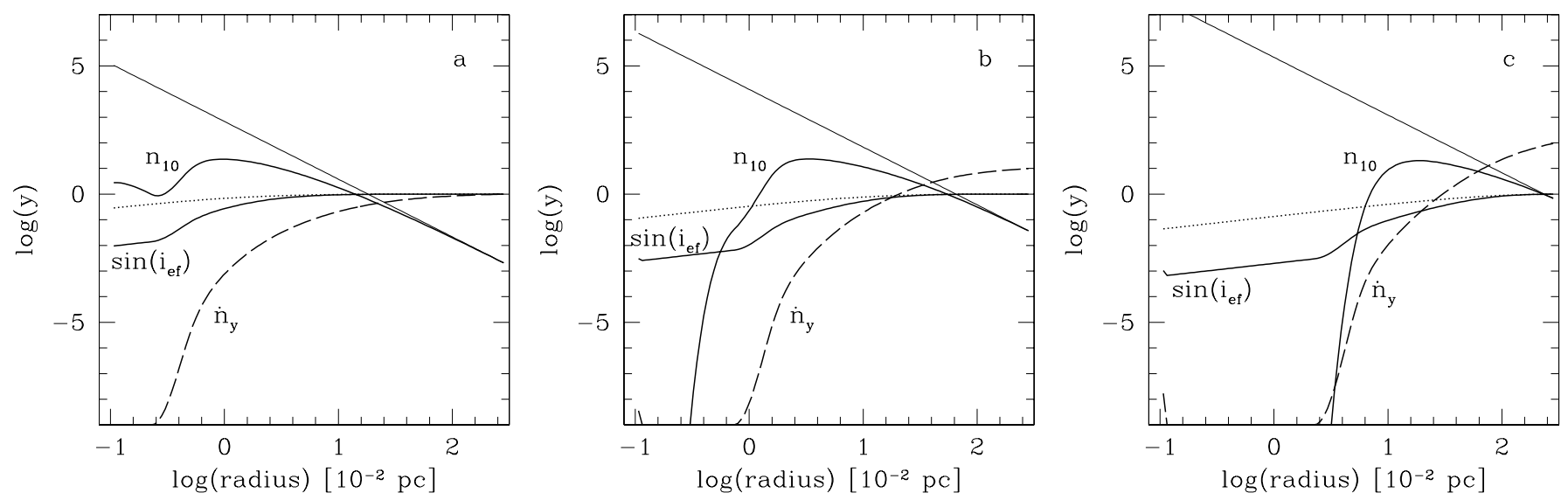

Fig. 3. The radial dependence of the stellar density $n_{10}, \sin \left(i_{\mathrm{ef}}\right)$ and stellar flow $\dot{n}_{y}$ for different black hole masses: a) $M_{\mathrm{BH}}=$ $10^{7} M_{\odot}$, b) $M_{\mathrm{BH}}=10^{8} M_{\odot}$, and c) $M_{\mathrm{BH}}=10^{9} M_{\odot}$. Thin continuous line shows the $n_{10}$ distribution in the absence of stellar collision effect.

MBH (Murphy et al. 1991). The application of a spherical model to the stellar dynamics in BELRs is questionable, because it completely ignores the interaction of stars with the AD. Our model of stellar dynamics includes both interactions which together determine the correct spatial and kinematical distribution of stars and we can now built a model of the BELR based on that knowledge.

To define the physical nature of the BELR clouds, and the model of the star-disk interaction in more details, we refer to the paper by $\dot{Z}$ urek et al. (1994), where the structure of the plasma around the star moving through the disk is presented as a combination of a hot bow-shock filled with hot plasma, and a more dense and cold wake, or tail, behind the star. We suppose that when the star comes out from the disk surface, the appearance of the bow-shock from the surface of the disk can be treated as a fireball, and the stellar wakes can be identified with the clouds of the BELR.

$\dot{Z}$ urek et al. (1994) assumed that the BELR clouds are actually wakes of matter, dragged by the stars from the disk. We accept this idea in a slightly changed variant, taking the view that the matter dragged from the disk creates a fireball, and the wakes contain the mass, lost by the stars when they cross the disk (both parts are the same to within an order of magnitude). As was described in the previous section, the fraction of mass lost by the star is proportional to the dissipated energy $\Delta E$, that is $\Delta M_{\mathrm{S}}=$ $0,01 q_{\mathrm{m}-2} \Delta E \mathrm{~d} M / \mathrm{d} E$, where $\mathrm{d} M_{\mathrm{s}} / \mathrm{d} E=R_{\mathrm{s}} /\left(G M_{\mathrm{s}}\right)$.

Taking $\Delta E$ from Eq. (6), one has

$M_{\mathrm{t}} \simeq \Delta M_{\mathrm{s}} \simeq 3.5 \times 10^{26} q_{\mathrm{m}-2} Q \Sigma_{4} r_{\mathrm{s}}^{3} m_{\mathrm{s}}^{-1} m_{8} r_{-2}^{-1} \phi_{1} \mathrm{~g}$,

where $q_{\mathrm{m}-2}=1$ means that we assume that $1 \%$ of the star's energy dissipated in the disk is used for the mass loss of the star.

As the balance of forces acting on the wakes depends on the parameters of AGNs and is model-dependent, we assume here the simplest case: the wakes follow the stars. Let the wakes be cylinders with lengths approximately equal to the height of the stellar orbit above the disk, $l_{\mathrm{t}} \sim R \sin \left(i_{\mathrm{ef}}\right)$, and let the radii of the cylinders be close to the stellar radius, $r_{\mathrm{t}} \sim r_{\mathrm{s}} R_{\odot}$ (this last suggestion may require the presence of a hot corona to provide the cold gas confinement). Then the mean particle density in the cylinder is

$n \simeq M_{\mathrm{t}} /\left[l_{\mathrm{t}} \pi r_{\mathrm{s}}^{2} \sin \left(i_{\mathrm{ef}}\right) m_{\mathrm{p}}\right]$,

or numerically

$n \simeq 3.8 \times 10^{11} q_{-2} Q \Sigma_{4} r_{\mathrm{s}} m_{\mathrm{s}}^{-1} m_{8} r_{-2}^{-2} \phi_{1} /\left(\sin i_{\mathrm{ef}}\right) \mathrm{cm}^{-3}$,

and the column density is

$N \simeq 6.5 \times 10^{22} q_{-2} Q \Sigma_{4} r_{\mathrm{s}} m_{\mathrm{s}}^{-1} m_{8} r_{-2}^{-2} \phi_{1} /\left(\sin i_{\mathrm{ef}}\right) \mathrm{cm}^{-2}$.

The covering factor $(\mathrm{CF})$ of a single cloud (along the disk radius) is

$\Omega=2 R_{\mathrm{s}} l_{\mathrm{t}} / 4 \pi R^{2} \simeq 3.7 \times 10^{-7} r_{\mathrm{s}} \sin i_{\mathrm{ef}} / r_{-2}$,

and the total covering factor of many clouds in a spherical layer from $R$ to $R+\mathrm{d} R$ (the differential $\mathrm{CF}$ ) is

$C F=4 \pi \Omega n_{\mathrm{s}} R^{2} \sin i_{\mathrm{ef}} \mathrm{d} R$,

where $n_{\mathrm{s}}$ is the stellar density (the number of clouds per unit volume is close to the stellar number density because we assume the life-time of a cloud is close to half the orbital period of the star: one star - one cloud). Taking into account that at small and intermediate inclination angles $\phi_{1} /\left(\sin i_{\mathrm{ef}}\right) \sim \sin i_{\mathrm{ef}}$, and that the flow of the ionizing photons is proportional to $\Phi_{\mathrm{i}} \sim 10^{22} m_{8} \dot{m} r_{-2}^{-2}$, one can see that the particle densities and the radiation flows are within the range of calculations of Korista et al. (1997) for the atlas of equivalent widths $(E W)$ of quasar broad emission lines. These calculations give $E W$ as a function of two parameters, $\Phi_{\mathrm{i}}$ and $n$, supposing that all the clouds have column densities $N=10^{23} \mathrm{~cm}^{-2}$ and the total covering factor of the clouds is $C F=1$. This permits us to calculate the profile of an emission line as

$$
\begin{aligned}
& I(V) \simeq K \int_{R_{\min }}^{R_{\max }} E W\left(\Phi_{\mathrm{i}}, n\right) \Omega n_{\mathrm{s}} R^{2} \sin i_{\mathrm{ef}} \mathrm{d} R \\
& \quad \times \iint\left\{C_{\mathrm{r}}(\varphi, \theta) /\left(v_{\mathrm{r}} \cos \varphi\right) \pi^{-1 / 2} D^{-1} \exp \left[-\left(v_{\mathrm{ch}} / D\right)^{2}\right]\right. \\
& \left.\quad \times \delta\left(V-v_{\mathrm{p}}-v_{\mathrm{ch}}\right)\right\} \mathrm{d} \varphi \mathrm{d} v_{\mathrm{ch}}
\end{aligned}
$$



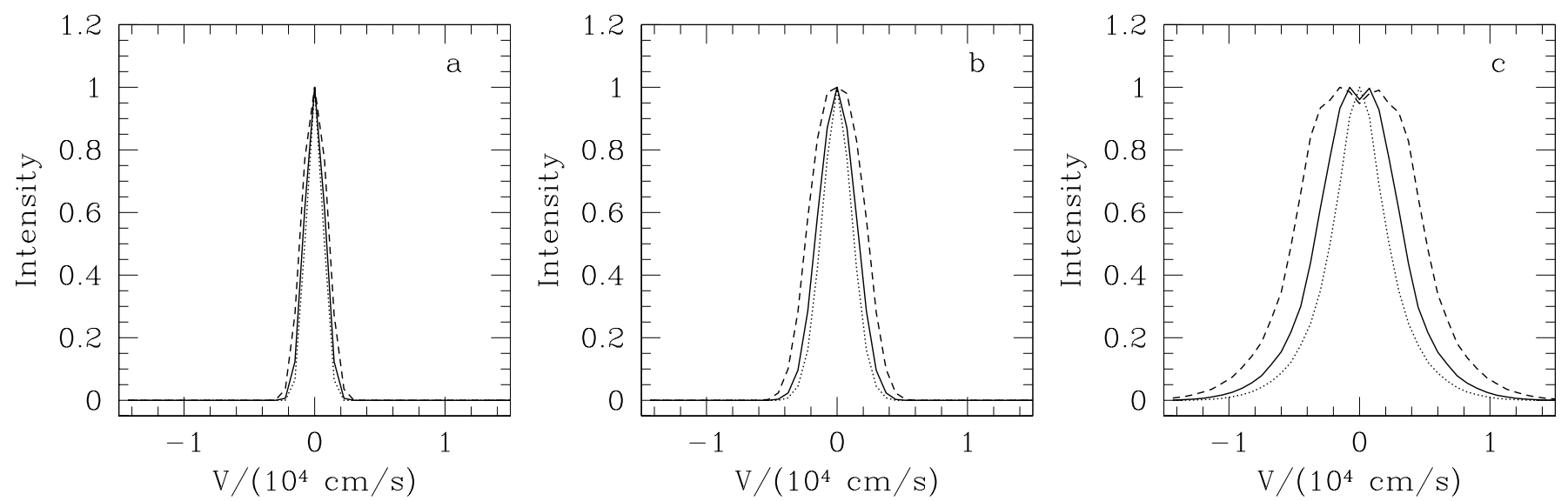

Fig. 4. $H_{\alpha}$ line profiles, calculated with $M_{\mathrm{BH}}=10^{7} M_{\odot}$ a), $M_{\mathrm{BH}}=10^{8} M_{\odot}$ b), and $M_{\mathrm{BH}}=10^{9} M_{\odot}$ c). Dotted, solid and dashed lines correspond to $\theta=10^{\circ}, 30^{\circ}$ and $60^{\circ}$.

where $V$ is the velocity projection along the line of sight, $K$ is a constant for a specific line, $E W\left(\Phi_{i}, n\right)$ is the $E W$ of the line calculated in Korista et al. (1997) as a function of $\Phi_{\mathrm{i}}$ and $n, \Omega$ is the coverage factor of a cloud at the distance $R, n_{\mathrm{S}}$ is the number density of stars (and so of the clouds), $C_{\mathrm{r}}$ is the relativistic correction, $v_{\mathrm{p}}(\varphi, \theta)=$ $v_{\mathrm{r}} \cos \varphi \sin \theta$ is the projection of the rotational velocity $v_{\mathrm{r}}$ onto the line of sight, ( $\varphi$ is the azimuth angle and $\theta$ is the angle between the line of sight and the axis of the disk), $v_{\mathrm{ch}}$ is the projection component of the chaotic velocity of stars with dispersion $D(R)=0.67 \times 10^{9}\left(M_{8} / r_{2}\right) \sin i_{\mathrm{ef}} \mathrm{cm} \mathrm{s}^{-1}$, where $M(R)$ is the total mass within radius $R$ and $\delta()$ is the delta-function.

Note that the velocity dispersion $D$ of the chaotic motion contains $\sin i_{\text {ef }}$, because the velocity dispersion is due to the distribution of the inclination angles and eccentricities around the effective values; we assume here that the distribution of the projection velocities is Gaussian in the coordinate system co-rotating with the AD.

We convolve the rotational velocity with the chaotic velocities of the stars; this means that the velocity of clouds contains both the rotational (disk) and chaotic velocity components of the stars. $R_{\min }$ and $R_{\max }$ in the integral limits are taken correspondingly as $0.001 \mathrm{pc}$ and $\left(m_{8} \dot{m}\right)^{1 / 2} \mathrm{pc}$, the last is approximately determined by the inner radius of the putative obscuring torus.

We performed the computations for the disk surface density profile of NT given by Eq. (7).

The results of the numerical calculations of the profiles of the $H_{\alpha}$ line, normalized to 1 at the maximum, are presented in Fig. 4. Each panel contains three profiles, corresponding to different inclination angles of the line of sight to the axis of the AD: $\theta=10^{\circ}, 30^{\circ}$ and $60^{\circ}$. The general shapes of the profiles are determined by the combination of a broad part, which depends mostly on the rotation of the inner region of the disk, and a more narrow central part, which is determined mostly by stellar chaotic motions in the outer parts of the system.

In Fig. 4(a-c) we show line profiles for the Eddington mass inflow $(\dot{m}=1)$ and for different masses of the $\mathrm{MBH}$, $m_{8}=0.1,1,10$, which would correspond to a Narrow-Line Seyfert 1 galaxy (NLS1), a Seyfert galaxy and a quasar, respectively. Our calculations support the idea that the narrow line Seyfert 1 galaxies are those with small MBH mass and almost Eddington mass inflow; or they can be objects with intermediate $\mathrm{MBH}$ mass and with a mass inflow less than the Eddington one, seen at an angle $\theta<30^{\circ}$.

Our theoretical consideration of the stellar distribution above the $\mathrm{AD}$ gives reasonable line profiles, supporting the idea that the emitting clouds are somehow related to the stars. They can be the wakes, following the stars after crossing the disk, or/and some gas envelopes of the stars themselves, which are disturbed by disk-crossing and by close passages of other stars. The mean distance between the stars at stellar density $n_{\mathrm{s}} \sim 10^{9} \mathrm{pc}^{-3}$ is $\mathrm{d} r \sim 10^{-3} \mathrm{pc}$, and many stars pass at even smaller distances, exciting each other by tidal forces and tangential collisions. However, in the present paper we used the specific model of clouds as the stellar wakes.

In our model the covering factor of the clouds along the AD plane became higher than 1 at large radii, so the spectrum of ionizing radiation has to be changed in the outer parts of the BELR. Of course, our model calculations are preliminary and rough, and a more detailed model has to be considered for the sake of comparisons with the observed profiles. But the common property following from the physical sense of our approach, viz. that the wings of the profiles should be more variable than the central peaks, seems to be confirmed by reverberation investigations.

\section{Variability}

In the present section we will estimate the level of variability driven by the stars. Obviously, the interpretation of the variability demands the calculation of rather complicated models, but here we will make a simple analysis of the variability expected from the inelastic processes of star-disk and star-star interactions. We consider two types of processes, connected with the energetic events of 
s-d and s-s collisions: flashes of UV radiation and X-ray flares. We consider a very simple model: let every event produce a fireball, filled with matter dragged from the disk (s-d crossing) or from stars (s-s collision), and expanding in a hot coronal medium. When the fireball becomes Compton-transparent, it produces a UV flare. The radiation of the flare interacts with the hot electrons of the corona above the disk, thus producing a flare of the up-scattered X-radiation.

The real picture is of course much more complicated. The fireball radiation spectrum evolves; quickly expanding fireballs create blast waves in the environment, and the bow shock can be accelerated when propagating from the disk surface into the corona with diminishing density. It is possible also that the matter of the fireball is heated later on to the coronal temperature, thus creating a transient patchy corona. The undisturbed, steadystate corona (without any influence of stars) can extend up to $R=300 R_{\mathrm{g}}$ (Kawaguchi et al. 2001); the patchy corona created by s-d interactions can extend to even larger distances.

All these processes require a more detailed description, but here we consider the simplest one, based on the simple picture of fireballs.

\subsection{The star-disk events}

When a star comes out of the disk, it is preceded by a bow-shock, accompanied by a fountain of matter from the disk. The total amount of gas thrown out above the disk consist of two parts: $\Delta M_{\mathrm{d}}$ is the mass dragged out from disk by the star, and $\Delta M_{\mathrm{s}}$ is the mass lost by the star.

We assume that the fireball is created by the first mass, and that the second mass creates the wake following the star and showing up as a BLER cloud, as was discussed in the previous section.

Then the mass of the fireball is $M_{\mathrm{f}}=\Delta M_{\mathrm{d}}=$ $q_{1} \pi R_{\mathrm{s}}^{2} \Sigma_{\mathrm{d}}$, where $q_{1} \leq 1$; numerically

$M_{\mathrm{f}} \simeq 1.6 \times 10^{26} q_{1} r_{\mathrm{s}}^{2} \Sigma_{4} \mathrm{~g}$.

Let the fireball contain the energy deposited by the star into the disk matter,

$\Delta E \simeq 0.7 \times 10^{44} Q r_{\mathrm{s}}^{2} \Sigma_{4} m_{8} r_{-2}^{-1} \phi(i) /(\cos i) \mathrm{erg}$.

We assume that the fireball expands and produces the flare when it becomes transparent, that is optically thin relative to the electron scattering. At this moment its radius fits the relation $R_{\mathrm{f}} \rho \sigma=1$, where $\rho=M_{\mathrm{f}} /\left(4 / 3 \pi R^{3}\right)$ is the density of the fireball, and $\sigma \simeq 0.4 \mathrm{~cm}^{2} \mathrm{~g}^{-1}$ is the Thomson scattering cross section per gram of the ionized cosmic plasma. Then the radius of the fireball at the moment of the flare is

$R_{\mathrm{f}} \simeq\left(M_{\mathrm{f}} \sigma_{T} / 4\right)^{1 / 2} \simeq .4 \times 10^{13}\left(q_{1} \Sigma_{4}\right)^{1 / 2} r_{\mathrm{s}} \mathrm{cm}$.

The density at the moment of the flare is

$\rho_{\mathrm{f}} \simeq M_{\mathrm{f}} /\left(4 / 3 \pi R_{\mathrm{f}}^{3}\right) \simeq 6.3 \times 10^{-13} q_{1}^{-1 / 2} r_{\mathrm{s}}^{-1} \Sigma_{4}^{-1 / 2}$, so the particle density is

$n=3.2 \times 10^{11} q_{1}^{-1 / 2} r_{\mathrm{s}}^{-1} \Sigma_{4}^{-1 / 2} \mathrm{~cm}^{-3}$,

and the column density is close to $N_{\mathrm{H}}=10^{24} \mathrm{~cm}^{-2}$.

The temperature in the fireball changes due to its expansion approximately as

$T_{\mathrm{f}} \simeq\left[q_{1} \Delta E /\left(4 / 3 \pi a R^{3}\right)\right]^{1 / 4} \mathrm{~K}$,

where $a=7.6 \times 10^{-15} \mathrm{erg} \mathrm{cm}^{-3} \mathrm{~K}^{-4}$, and $R$ is the radius of the expanding fireball.

At the moment of the flare, the temperature is therefore

$T_{\mathrm{f}} \simeq .8 \times 10^{5} Q_{\mathrm{T}} r_{\mathrm{s}}^{-1} \Sigma_{4}^{-1 / 2} m_{8}^{1 / 4} r_{-2}^{-1 / 4} \phi 1^{1 / 4} \mathrm{~K}$,

where $Q_{\mathrm{T}}=q_{1}{ }^{-3 / 8} Q^{1 / 4}$, and the spectral maximum of the flare is in the far UV-band,

$\nu \sim 5 \times 10^{15} \mathrm{~Hz}$.

The estimate of the minimum duration of the flare (assuming that the radiation leaves the fireball when the latter becomes Compton-thin) is

$\Delta t_{\mathrm{f}} \leq 2 R_{\mathrm{f}} / c \simeq .3 \times 10^{3} q_{1} \Sigma_{4}^{1 / 2} r_{\mathrm{s}} \mathrm{s}$,

and the average luminosity during the flare is

$L_{\mathrm{f}}=\Delta E_{\mathrm{f}} / \Delta t_{\mathrm{f}} \leq 3 . \times 10^{41} q r_{\mathrm{s}} \Sigma_{4}^{1 / 2} m_{8} r_{-2}^{-1} \phi_{1} \mathrm{erg} \mathrm{s}^{-1}$,

where $q=q_{1} Q$.

In the NT disk we have

$L_{\mathrm{f}} \simeq 0.5 \times 10^{43} q r_{\mathrm{s}} m_{8}^{9 / 8} \alpha_{-2}^{-2 / 5}\left(\dot{m}_{1}\right)^{7 / 20} r_{-2}^{-11 / 8} \phi_{1} \mathrm{erg} \mathrm{s}^{-1}$.

The real duration of the flare is rather longer, and the luminosity is less than in these estimates. When the temperature of the fireball becomes lower than $10^{5} \mathrm{~K}$, the plasma in the fireball partly recombines, which increases the opacity to above its Thomson value. Besides, if the fireball expands with a velocity close to the sound velocity $V_{\mathrm{f}} \simeq\left(k_{\mathrm{B}} / m_{\mathrm{p}} T_{\mathrm{f}}\right)^{1 / 2} \simeq 3 \times 10^{6} \mathrm{~cm} \mathrm{~s}^{-1}$, the time of expansion is $t_{\mathrm{ex}} \simeq R_{\mathrm{f}} / V_{\mathrm{f}} \simeq 10^{6} \mathrm{~s}$. So, rough estimates show that the characteristic duration of the flare is in between the two limits

$3 \times 10^{2} q_{1} \Sigma_{4}^{1 / 2} r_{\mathrm{s}}<\Delta t_{\mathrm{f}}(\mathrm{s})<10^{6} q_{1} \Sigma_{4}^{1 / 2} r_{\mathrm{s}} /\left(T_{\mathrm{f} 5}\right)^{1 / 2}$.

Now we estimate the combined effect of all star-disk events.

It has to be noted here that the results of model calculations of luminosity and variability, connected with the star-disk interaction, depend strongly on the values of the rates of energy change, estimated by Eqs. (14) and (6). In principle, these estimates can be increased, because the coefficient $Q$ in $\mathrm{d} E^{(\mathrm{sd})} / \mathrm{d} t$ is unspecified, and $\mathrm{d} E^{(\mathrm{ss})} / \mathrm{d} t$ can be increased, e.g. due to collective processes in the flat distribution of stars. The same effect of increasing luminosity is expected if the equilibrium $\mathrm{d} E^{(\mathrm{sd})} / \mathrm{d} t=\mathrm{d} E^{(\mathrm{ss})} / \mathrm{d} t$ is not reached, due to larger values of $\sin \left(i_{\mathrm{ef}}\right)$. Because of this, we calculated all quantities of interest (see below) for our 
reference models with $m_{8}=0.1,1,10$ for two cases: 1$)$ energy change rates given by Eqs. (14) and (6); 2) energy change rates increased by a factor of 5 .

The mean luminosity provided by star-disk interaction in the ring between $R$ and $R+\mathrm{d} R$ is $\mathrm{d} L=\Delta E_{\mathrm{f}} \mathrm{d} \nu_{\mathrm{sd}}$, with $\mathrm{d} \nu_{\text {sd }}$ from Eq. (42):

$$
\begin{aligned}
\mathrm{d} L \simeq & 2 \times 10^{43} Q \Sigma_{4}\left[\dot{n}_{\mathrm{y}}^{r} /(\ln \Lambda)_{1}\right]^{1 / 2} \\
& \times r_{\mathrm{s}}^{2} m_{\mathrm{s}}^{-1} m_{8}^{9 / 4} r_{-2}^{-11 / 4} \phi(i) \sin \left(i_{\mathrm{ef}}\right) \mathrm{d} r_{-2},
\end{aligned}
$$

and the total luminosity of the flares is

$L_{\mathrm{fT}}=\int L_{\mathrm{f}} \Delta t_{\mathrm{f}} \mathrm{d} \nu_{\mathrm{sd}}=\int \Delta E_{\mathrm{f}} \mathrm{d} \nu_{\mathrm{sd}}$

From calculations of the reference models with $m_{8}=$ $0.1,1,10$, we have the total UV-flare luminosities (in units of $10^{43} \mathrm{erg} \mathrm{s}^{-1}$ ) in the first case $L_{\mathrm{fT}} \simeq 0.02,0.07,0.39$, and for the second case $(\mathrm{d} E / \mathrm{d} t 5$ times larger $): L_{\mathrm{fT}} \simeq$ $2.2,29,7.4$.

One can see that in the second case the total luminosity of flares resulting in the s-d interactions is comparable to the variable fraction of the UV luminosity.

The average number of simultaneous flares in every $\mathrm{d} r$ - ring is $\mathrm{d} L / L_{\mathrm{f}}$, so the total number of coexisting flares is

$N=\int \Delta t_{\mathrm{f}} \mathrm{d} \nu_{\mathrm{sd}}$

For the three MBH masses $\left(m_{8}=0.1,1,10\right)$, we have (with $\left.\Delta t^{\mathrm{min}}\right)$ in the first case $N \simeq 3.6,5.7,100$, and in the second case $N \simeq 17,73,300$.

The weighted luminosity of a flare is $\tilde{F}_{\mathrm{f}}=L_{\mathrm{fT}} / N$, and the mean-square deviation of the luminosity due to chaotic variability is

$D L_{\mathrm{sd}}=\tilde{F}_{\mathrm{f}} N^{1 / 2}=L_{\mathrm{fT}} / N^{1 / 2}$.

Corresponding values for the three $\mathrm{MBH}$ masses are in the first case $D L / L_{\mathrm{f}}=1 / N^{1 / 2} \simeq 0.5,0.4,0.1$, and in the second case $D L / L_{\mathrm{f}} \simeq 0.24,0.12,0.06$; so, the variability amplitude diminishes with increasing luminosity of AGNs.

\subsection{The star-star events}

Similarly we can estimate the variability connected with the star-star contact collisions. We assume that the average mass-loss per collision is

$M_{\mathrm{f}} \sim 0.1 \zeta_{-1} M_{\mathrm{s}}=2 \times 10^{32} \zeta_{-1} m_{\mathrm{s}} \mathrm{g}$.

The parameters of the s-s fireballs (the total energy, the radius of the fireball, the temperature of UV radiation, the minimum and maximum duration of the UV flare are:

$$
\begin{aligned}
& \Delta E=0.45 \times 10^{50} \zeta_{-1} m_{\mathrm{s}} m_{8} r_{-2}^{-1} \sin ^{2}\left(i_{\mathrm{ef}}\right) \mathrm{erg}, \\
& R_{\mathrm{f}} \simeq\left(M_{\mathrm{f}} \sigma_{\mathrm{T}} / 4\right)^{1 / 2} \simeq 1.1 \times 10^{16} m_{\mathrm{s}}^{1 / 2} \zeta_{-1}^{1 / 2} \mathrm{~cm}, \\
& T_{\mathrm{f}} \simeq 3.6 \times 10^{4} \zeta_{-1}^{1 / 4} m_{\mathrm{s}}^{-1 / 4} m_{8}^{1 / 4} r^{-1 / 4} \sin ^{2} i_{\mathrm{ef}} \mathrm{K} \\
& \Delta t_{\mathrm{f}}^{\min } \geq 2 R_{\mathrm{f}} / c=0.7 \times 10^{6} m_{\mathrm{s}}^{1 / 2} \zeta_{-1}^{1 / 2} \mathrm{~s} \\
& \Delta t_{\mathrm{f}}^{\max } \leq 2 R_{\mathrm{f}} / V_{\text {sound }} \simeq 3 \times 10^{9} \mathrm{~s} .
\end{aligned}
$$

The corresponding average luminosity is given by

$$
\begin{aligned}
& L_{\mathrm{f}}^{\max } \leq .64 \times 10^{44} \zeta_{-1}^{1 / 2} m_{\mathrm{s}}^{1 / 2} m_{8} r^{-1} \sin ^{2} i_{\mathrm{ef}} \mathrm{erg} \mathrm{s}^{-1} \\
& L_{\mathrm{f}}^{\min } \geq 2 \times 10^{41} \zeta_{-1}^{1 / 2} m_{\mathrm{s}}^{1 / 2} m_{8} r^{-1} \sin ^{2} i_{\mathrm{ef}} \mathrm{ergs}^{-1}
\end{aligned}
$$

By definition, the column density of the fireball at the moment of the flare is $N=R n \sim 10^{24} \mathrm{~cm}^{-3}$, so $n \simeq$ $10^{9} \mathrm{~cm}^{-3}$.

Taking $\mathrm{d} \nu_{\mathrm{ss}}$ from Eq. (31), we have a number of simultaneous flares $N=\int \Delta t_{\mathrm{f}} \mathrm{d} \nu_{\mathrm{ss}}$, which is typically less than 1 . This means that s-s events lead to even lower average luminosity than s-d ones, though the amplitude of the s-s flares are larger.

\subsection{The relation of the $U V$ and $X$-ray events and the frequency dependence}

Of course, the variability connected with stars may not be the only mechanism operating in AGN. Their variability can be mostly driven by the variable (unstable) accretion rate onto the $\mathrm{MBH}$. The process of accretion itself is rather complicated, and the theory develops quickly (see the recent review in Park \& Ostriker 2001). The variability observations show some common properties of the variability of the AGNs and the stellar-mass Galactic black hole candidates (Utley \& McHardy 2001; Chiang et al. 2000), and for that class of objects the model of distributed flares has been considered (Galeev et al. 1979; Czerny \& Lehto 1997; Poutanen \& Fabian 1999). On the other hand, it has been shown that UV and X-ray variability of AGNs cannot be explained with models assuming simple reprocessing of the X-ray emission of a variable point source placed at the symmetry axis above AD. Is it possible that s-d collisions can work in AGNs instead of the magnetic flares supposed to exist in the Galactic black holes?

From the rough estimates provided in the previous subsection one can conclude that the s-d interaction can lead to a noticeable variability amplitude only if both rates of energy dissipation - due to s-d and s-s interactions - are enhanced several times in comparison to our basic estimates. This can possibly be achieved with a more realistic distribution of stellar masses. An increase might also come due to unusual properties of the stars in the very compact stellar clusters. Deviations from the equilibrium conditions (which was assumed by us for the approximate solutions) also promote larger values of $\sin \left(i_{\mathrm{ef}}\right)$ and, as a consequence, lead to more power radiated from s-d interactions.

Obviously, the UV events are divided into two types: those connected with s-d and with s-s interactions. The relations of the two types of interactions to the X-ray events are different.

In the s-d case, the UV flares occur at relatively small heights above the $\mathrm{AD}$, which are close to the fireball size, $h \sim 0.39 \times 10^{13}\left(q_{1} \Sigma_{4}\right)^{1 / 2} r_{\mathrm{s}} \mathrm{cm}$. The time for reaching this distance is $t \sim R_{\mathrm{f}} / V_{\mathrm{f}}$, where $R_{\mathrm{f}}$ and $V_{\mathrm{f}}$ are the size of the fireball and the velocity of its expansion, and the time 
from the appearance of the blast wave to the maximum of the UV luminosity is $t \sim 0.39 \times 10^{4}\left(q_{1} \Sigma_{4}\right)^{1 / 2} r_{\mathrm{s}}$ s. The size of the region of up-scattering is about the coronal scale height, around $10^{13}-10^{14} \mathrm{~cm}$, which leads to a time-lag between UV and X-ray flares of about $\Delta t_{\mathrm{f}} \sim 10^{3}-10^{4} \mathrm{~s}$.

In the s-s case, most events occur at large heights above the disk, so the fraction of photons reflected by the corona is small, and the duration of Compton-scattered X-ray events, as well as the time-lag, are much longer than in the case of the s-d event.

Using the estimates from the previous section, we can consider the amplitude-frequency relations following from star-disk and star-star dissipative interactions. In Fig. 5 we plot the dependences of the UV flare luminosity $L_{\mathrm{f}}=\Delta E_{\mathrm{f}}(R) / \Delta t_{\mathrm{f}}(R)$, divided by frequency $\mathrm{d} \nu(R)$ on the frequency. We plot both $L_{\mathrm{fss}} / \mathrm{d} \nu_{\mathrm{ss}}$ and $L_{\mathrm{fsd}} / \mathrm{d} \nu_{\mathrm{sd}}$ in the same figure using the above-defined dependence of both values on radius and replacing the radial coordinate with the frequency coordinate where the frequency-radius relation is taken from the determined characteristic frequency of events at a given radius. Let us call the dependence "frequency-amplitude dependence" (FAD). Note that the FAD cannot be strictly compared to the power density spectrum (PDS), observed in the UV and X-ray bands (e.g. McHardy \& Czerny 1987; Chiang et al. 2000). These PDS are obtained by Fourier transformation of the light curves, which are usually measured in frequency bands about $10^{-7}-10^{-4} \mathrm{~Hz}$ and show a sort of red noise, that is $P D S \propto \nu^{-p}$ with $p \sim 1-2$; sometimes $p \sim 0$ is found at the low-frequency limit. The FAD spectrum shown in Fig. 5 has a different meaning: it is just the dependence of the flare luminosity per frequency interval on the frequency of the events. Besides, we recall that we use the equal stellar mass and other approximations, so the real slopes of the curves could be steeper and the curves could cover a broader frequency range. Nevertheless, some general prediction about PDS can be derived from our calculations:

i) the PDS of AGNs can be divided into two parts: fast variability (on time scales from hundreds of seconds to about a year, which is connected with the s-d processes) and slow variability (on time scales from several years to thousands of years, connected with the s-s collisions);

ii) the PDS slope can change abruptly somewhere in between the fast and slow part, and the slow variability part of the PDS has a larger amplitude. This conclusion seems to be in agreement with the results of long-time observations in X-ray band (Markowitz \& Edelson 2001), and it is definitely supported by long-time observations in the optical band (Czerny et al. 1999; Czerny et al. 2001);

iii) the differences of PDS between the slow and fast parts may depend on the AGN luminosity and other parameters.

Our preliminary conclusions are that the presence of a CSC in AGNs does not contradict the observations, the CSC can contribute to AGN variability in some cases, and further comparison of variability of AGNs and Galactic black holes is needed.

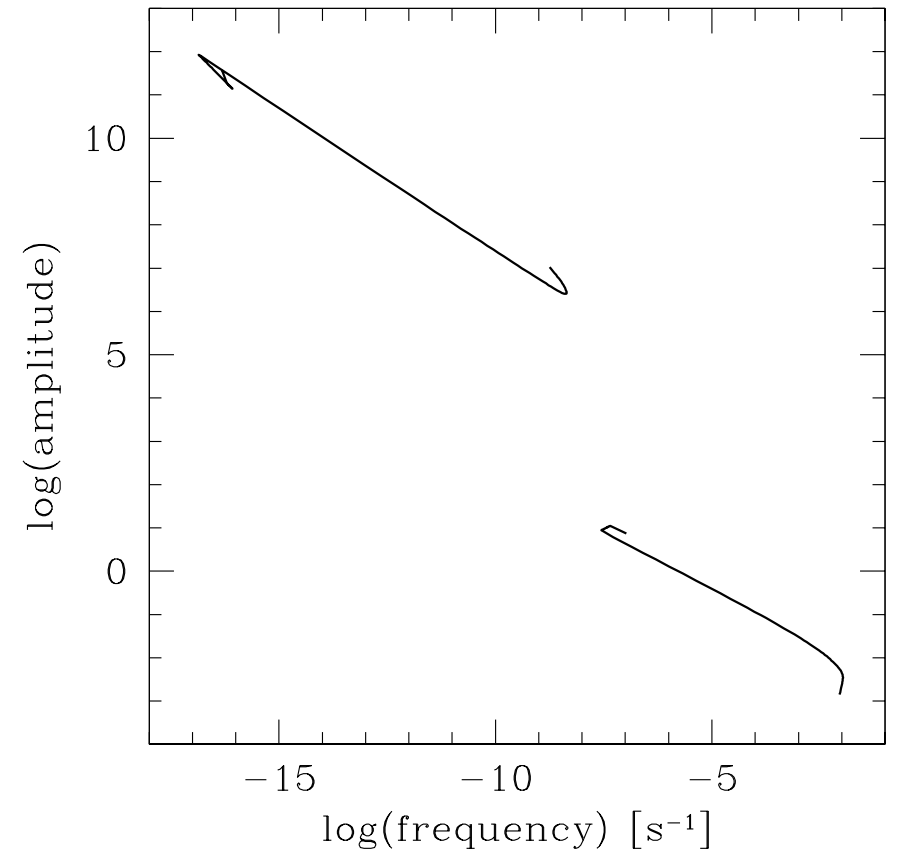

Fig. 5. The frequency-amplitude dependence, including s-d (high-frequency) and s-s (low frequency) interactions.

\section{Discussion and conclusions}

The role of the compact stellar cluster in the creation and evolution of AGN is still poorly understood. We suppose that this role is very important, so that the AGN phenomenon can be considered as a result of interactions of three main physical subsystems: the $\mathrm{CSC}, \mathrm{MBH}$ and gas subsystem. We call this approach the interacting subsystems theory (Vilkoviskij et al. 1999).

Our model of the CSC-disk interaction, though very approximate, is the first one which treats star-star and star-disk interactions jointly, so it can serve as a starting point for considering some new physics in AGN problems.

We showed that the star-disk interaction tends to order stellar orbits, dragging them to the disk plane and making them circular, but the star-star gravitational interaction scatters these orbits and prevents them from being incorporated into the disk. We assume equilibrium of the two processes and use a simple inflow model instead of analyzing the kinetic equations. The other simplification was the assumption of the equal stellar masses, which is acceptable as a first step only.

Our results can be compared with the results of Rauch (1995, 1999). In the last work he calculated numerically the evolution of the spherically-symmetric stellar cluster with stellar collisions, but without s-d interactions. He finds that the internal stellar density distribution achieves a quasi-stationary state in the late stages. Our calculations of the radial stellar density distribution with direct stellar collisions and s-d interactions are in qualitative agreement with those of Rauch (1999).

On the other hand, our calculations of the shape of the stellar density distributions in the central parts of AGNs, are naturally different to the results of Rauch (1995), 
where he considered the evolution of individual stellar orbits crossing the disk (without s-s interaction). He calculated the evolution of the stellar system, which surrounds the $\mathrm{AD}$ in the first moment, and showed that the evolution leads to the completely flat system, incorporated into the disk plane in the final stage. In contrast, we take into account (besides the s-d interaction) both elastic and non-elastic star-star interactions. This leads to a quasistationary distribution of stars, which is flat only in the central part of the disk.

The determination of a more realistic stellar distribution in the innermost part of the central cluster allowed us to consider some consequences of the complex system of AGN, containing $\mathrm{MBH}, \mathrm{AD}$ and CSC.

We showed that the simple model of stellar wakes accompanying the s-d interactions gives profiles of broad emission lines similar to those observed, providing a reasonable solution for the puzzles of both generation and dynamics of the BELR clouds. We used even further simplified models for estimates of the variability connected with s-d and s-s interactions, and we come to the preliminary conclusion that a noticeable role of the stars in the variability is possible, but only if both dissipative and scattering interactions of stars are enhanced relative to our preliminary estimates. The variability produced by stars is naturally divided into a fast and a slow part, corresponding to s-d and s-s processes.

Of course, the main questions remains whether the stellar density of CSC in AGNs is large enough for all the effects discussed to proceed efficiently. Here we assumed that the masses of CSC in the bright AGNs are about an order of magnitude larger than the MBH mass.

The real stellar density and masses of the CSC in AGNs are still unknown. In the paper of Schinnerer et al. (2001) the near-infrared high angular resolution speckle imaging of the central region of the Seyfert galaxy NGC 3227 was performed. The authors showed that the CSC do exist in the inner $300 \mathrm{pc}$, and that in the innermost (unresolved, $<70 \mathrm{pc}$ ) part there is the even more dense core of the stellar cluster, which contributes less to the luminosity, but contains $85 \%$ of the mass of the cluster. This result supports our leading idea of large enough masses of CSCs in AGNs. A fuller investigation of 112 Seyfert galaxies in $1.6 \mu \mathrm{m}$ with the Hubble Space Telescope was performed by Quillen et al. (2001). They showed that there are unresolved sources in all Seyfert galaxies with luminosities $L(1.6 \mu \mathrm{m}) \sim\left(10^{41}-10^{42}\right)$ erg s ${ }^{-1}$ correlating with $\mathrm{O}[\mathrm{III}] \lambda 5007$ and with hard $\mathrm{X}$-ray luminosities for Seyfert 1.0-1.9 sources. The luminosities are much lower in Seyfert 2.0 sources. Due to this trends the authors concluded that the unresolved sources are mostly of nonstellar (hot dust) origin; but we note that in our model the correlation of the AGN luminosity with the mass of the CSC is expected. More detailed observations are needed for solving the question about CSC masses in AGNs.

It follows from our models that the accretion disk itself can be created as the result of mass loss due to contact stellar collisions in the rotating, compact and massive
CSC. In this case the collision disruptions of stars can partly feed the accretion disk flow, and another part of this gas (the hot part) can support the outflow of the hot gas from AGNs, as was supposed by Vilkoviskij et al. (1999). This opens new possibilities for the more self-consistent models of the AGN structure, where the accretion disk, obscuring torus and polar outflow are created (partly at least) due to stellar collisions in the rotating CSC. The transfer of angular momentum from AD to the CSC also has to be taken into account.

One can imagine the following general picture of the AGN's duty cycle:

The bright phase of AGNs should be relatively short, about several times $10^{8}$ years, and usually the activity cycle consists of three phases. It starts with creating a new CSC in the dense gas cocoon around the MBH (say, generated by a galactic merger), which corresponds to the IR-bright IRAS galaxies (the first phase). In this phase a new CSC is formed due to the powerful star-burst in the galactic center, which leads to the creation of a new AD, thus initiating the star-disk dynamics, similar to what was considered by Rauch (1995).

The subsequent growth of the AD luminosity, the creation of the central flat stellar distribution, and the appearance of the polar jets and the hot-gas outflows make polar holes in the cocoon, and the second phase of activity sets in; the AGN looks like a typical QSO or Seyfert galaxy with an obscuring torus. Now in this phase most of the stars in the polar regions of the rotating CSC are exhausted, and stars cross mostly the periphery of the AD due to the diffusion into an effective loss-cone. The AD itself can be supported by stellar collisions in the rotating CSC, and our solutions approximate stellar dynamics in this phase. During approximately $10^{8}$ years the mass of the CSC and the stellar inflow will gradually decrease, thus leading to the third phase with diminished activity, corresponding to the weak Seyferts and LINERs (galaxies with Low Ionization Narrow Emission Region).

Therefore, the results of our paper correspond to the second phase, the "adult and strong" age of an AGN.

It is clear that more detailed model calculations are required. Our description of star-star interaction in the nonspherical, flat stellar system is very rough and the results should be verified with $n$-body simulations. Advanced models should also take into account the distribution of stellar masses and a more accurate description of the accretion disk. New achievements in theory and observations of AGN show that the accretion flow is possibly a combination of a geometrically thin cold disk in the outer part, and an advection-dominated accretion flow (ADAF) in the central part of the disk in some cases (Ichimaru 1977; Rees et al. 1982; Narayan \& Yee 1994; Abramowicz et al. 1995; Loska \& Czerny 1997), with the hot corona still present above the cold disk (Esin et al. 1997; Esin et al. 1998; Witt et al. 1997).

However, even the approximate results presented here show that compact and massive stellar clusters in the 
centers of AGNs can play an important role in the creation and physical properties of the AGN engine.

Acknowledgements. E.Y.V. is grateful to Suzy Collin for many discussions, initiating this work, and to CAMK for the kind hospitality permitting to write this work.

The work was supported by grant No. 2P03D01816 of the Polish State Committee for Scientific Research, and partly by the INTAS grant No. 96-0328.

\section{References}

Abramowicz, M. A., Chen, X., Kato, S., Lasota, J.-P., \& Regev, O. 1995, ApJ, 438, L37

Alexander, T., \& Netzer, H. 1994, MNRAS, 270, 781

Alexander, T., \& Netzer, H. 1997, 284, 967

Artymowicz, P., Lin, D. C. N., \& Wampler, E. J. 1993, ApJ, 409, 592

Bahcall, J. N., \& Wolf, R. A. 1976, ApJ, 209, 214

Bisnovatyi-Kogan, G. S. 1978, Astron. Lett., 4, 130

Chiang, J., Reynolds, C. S., Blaes, O. M., et al. 2000, ApJ, 528,292

Collin, S., \& Hure, J.-M. 2001, A\&A, 372, 50

Collin, S., \& Zahn, J.-P. 1999, A\&A, 344, 433

Czerny, B., \& Lehto, H. J. 1997, MNRAS, 285, 365

Czerny, B., Doroshenko, V. T., Nikołajuk, M., et al. 2001, MNRAS, submitted [astro-ph/0110708]

Czerny, B., Schwarzenberg-Czerny, A., \& Loska, Z. 1999, MNRAS, 303, 148

Dumont, A.-M., \& Collin-Souffrin, S. 1990, A\&A, 229, 302

Edvards, A. S. 1980, MNRAS, 190, 757

Esin, A. A., McClintock, J. E., \& Narayan, R. 1997, ApJ, 489, 865

Esin, A. A., Narayan, R., Cui, W., Grove, J. E., \& Zhang, S. N. 1998, ApJ, 505, 854

Galeev, A. A., Rosner, R., \& Vaiana, G. S. 1979, ApJ, 484, 383

Hara, T. 1978, Progr. Theor. Phys., 60, 711

Hills, J. G. 1975, Nature, 256, 295

Ichimaru, S. 1977, ApJ, 214, 840

Karas, V., \& Vokrouhlicky, D. 1993, MNRAS, 265, 365

Kawaguchi, T., Shimura, T., \& Mineshige, S. 2001, ApJ, 546, 966

Kazans, D. 1989, ApJ, 347, 74

Korista, K., Baldwin, G., Ferland, G., \& Verner, D. 1997, ApJS, 108, 401

Loska, Z., \& Czerny, B. 1997, MNRAS, 284, 946
Markowitz, A., \& Edelson, R. 2001, ApJ, 547, 684

McHardy, I. M., \& Czerny, B. 1987, Nature, 325, 696

Murphy, B. W., Cohn, H. N., \& Durisen, R. H. 1991, ApJ, 370, 60

Narayan, R., \& Yee, I. 1994, ApJ, 428, L13

Novikov, I., \& Thorne, K. S. 1973, in Black Holes, ed. C. de Witt, \& B. S. de Witt (Gordon \& Breach, NY), 436

Ostriker, J. P. 1983, ApJ, 273, 99

Park, M. G., \& Ostriker, J. P. 2001, ApJ, 549, 100

Peebles, P. J. E. 1972, ApJ, 178, 371

Penston, M. V. 1988, MNRAS, 233, 601

Poutanen, J., \& Fabian, A. C. 1999, MNRAS, 306, L31

Quillen, A. C., McDonald, C., Alonso-Herrero, A., et al. 2001, ApJ, 547, 129

Rauch, K. P. 1995, MNRAS, 275, 628

Rauch, K. P. 1999, ApJ, 514, 725

Rees, M. J., Begelman, M. C., Blandford, R. D., \& Phinney, E. S. 1982, Nature, 295, 17

Saslaw, W. C. 1966, PASP, 85, 5

Schinnerer, E., Eckart, A, \& Taccony, E. J. 2001, ApJ, 549, 254

Scoville, N., \& Normann, C. 1988, ApJ, 332, 163

Scoville, N., \& Normann, C. 1995, ApJ, 541, 510

Shakura, N. I., \& Sunyaev, R. A. 1973, A\&A, 24, 337

Shklovskiy, I. S. 1964, Astron. Zh., 41, 801

Shull, J. M. 1983, ApJ, 264, 446

Subr, L., \& Karas, V. 1999, A\&A, 352, 452

Syer, D., Clarke, C. J., \& Rees, M. J. 1991, MNRAS, 250, 505

Turner, T. J., George, I. M., Nandra, K., \& Turcan, D. 1999, ApJ, 524, 667

Unno, W. 1971, PASP, 23, 123

Uttley, P., \& McHardy, I. M. 2001, MNRAS, 323, L26

Vilkoviskij, E. Y. 1975, Astron. Lett., 1, N7, 8

Vilkoviskij, E. Y., \& Bekbosarov, N. 1981, Izvestiya AN Kaz, 4,10

Vilkoviskij, E. Y. 1983, Astron. Lett., 9, N7, 405

Vilkoviskij, E. Y., Efimov, S. N., Karpova, O. G., \& Pavlova, L. A. 1999, MNRAS, 309, 80

Vokrouhlicky, D., \& Karas, V. 1993, MNRAS, 265, 365

Vokrouhlicky, D., \& Karas, V. 1998, MNRAS, 298, 53

Witt, H. J., Czerny, B., \& Życki, P. T. 1997, MNRAS, 286, 848

Zentsova, A. S. 1983, Ap\&SS, 95, 11

Żurek, W., Siemiginowska, A., \& Colgate, S. A. 1994, ApJ, 434,46 